TRANSACTIONS OF THE

AMERICAN MATHEMATICAL SOCIETY

Volume 354, Number 12, Pages 5087-5110

S 0002-9947(02)03054-4

Article electronically published on July 16, 2002

\title{
ON THE BLOW-UP OF HEAT FLOW FOR CONFORMAL 3-HARMONIC MAPS
}

\author{
CHAO-NIEN CHEN, L. F. CHEUNG, Y. S. CHOI, AND C. K. LAW
}

Dedicated to Shui-Nee Chow on the occasion of his 60th birthday

\begin{abstract}
Using a comparison theorem, Chang, Ding, and Ye (1992) proved a finite time derivative blow-up for the heat flow of harmonic maps from $D^{2}$ (a unit ball in $\mathbf{R}^{2}$ ) to $S^{2}$ (a unit sphere in $\mathbf{R}^{3}$ ) under certain initial and boundary conditions. We generalize this result to the case of 3-harmonic map heat flow from $D^{3}$ to $S^{3}$. In contrast to the previous case, our governing parabolic equation is quasilinear and degenerate. Technical issues such as the development of a new comparison theorem have to be resolved.
\end{abstract}

\section{INTRODUCTION}

In this paper, we investigate the finite time blow-up phenomenon for 3-harmonic map heat flow from a three-dimensional unit ball of the Euclidean space to the three-dimensional sphere. Let $D^{n}$ be a unit ball of $\mathbf{R}^{n}$ and let $S^{n}$ be the boundary sphere of $D^{n+1}$ in $\mathbf{R}^{n+1}$, both centered at the origin. With $0<\alpha<1, u_{0} \in$ $C^{2+\alpha}\left(\overline{D^{n}}, S^{n}\right)$, and $\left|u_{0}\right|=1$, we study the heat flow equations of the $p$-harmonic map

$$
u_{t}=\nabla \cdot\left(|\nabla u|^{p-2} \nabla u\right)+|\nabla u|^{p} u
$$

with the initial and boundary conditions

$$
\left\{\begin{array}{ll}
u(x, 0) & =u_{0}(x) \\
\left.u(\cdot, t)\right|_{\partial D^{n}} & =\left.u_{0}\right|_{\partial D^{n}}
\end{array} .\right.
$$

For (1.1), a $C^{1}$ solution is said to blow up in finite time if the maximal interval of existence is bounded; in other words, some of its derivatives become unbounded in finite time. The main result of this paper is to give a partial answer to the following question:

$\mathbf{P}(\mathbf{n}, \mathbf{p})$ : For the p-harmonic map heat flow, does blow-up occur in finite time? If so, for which $p$ and $n$, and for which initial data $u_{0}$ ?

1.1. A brief survey and an overview. This problem dates back to a surprising finite time blow-up theorem of Chang, Ding, and Ye 2] for the 2-harmonic map heat flow from the 2-ball to the 2-sphere for certain rotationally symmetric initial data. We note that a 2-harmonic map is traditionally called a harmonic map. Prior to their theorem, various finite time blow-up results were known for the harmonic map

Received by the editors June 25, 2001 and, in revised form, December 21, 2001.

2000 Mathematics Subject Classification. Primary 35K50, 35K65, 58E20.

Key words and phrases. p-harmonic maps, heat flow, blow up, maximum principle.

(C)2002 American Mathematical Society 
heat flow in cases the domain has dimension larger than two. One of the pioneering works in this direction was due to Coron and Ghidaglia [7] who established finite time blow-up theorems for the harmonic map heat flow from the $n$-ball to the $n$-sphere $(n \geq 3)$ for certain rotationally symmetric initial data. The finite time blow-up theorem for the harmonic map heat flow was a surprise, because prior to the work of Chang, Ding and Ye, the contrary was widely believed (see [2], p. 507). The special role played by harmonic maps on 2-dimensional domains is most succinctly summarized by the regularity theorem of Hélein [18, which states that all weak harmonic maps on surfaces are indeed smooth. In contrast to this beautiful result, only a partial regularity result can be proved for the weak harmonic map in higher dimensions 29], 12. These results give smoothness outside a set of zero $(n-2)$-dimensional Hausdorff measure, provided that the harmonic map is a minimizer.

The $p$-harmonic maps are the solutions of the Euler-Lagrange equations associated with the $p$-energy $E(f)$ defined by

$$
E(f)=\frac{1}{p} \int_{M}\left|d f_{x}\right|^{p},
$$

where $f$ is a $C^{1}$-map from a manifold $M$ to another manifold $N$. For $p>2$, the corresponding Euler-Lagrange equations form a degenerate elliptic system. The $p$-harmonic flow is the heat flow associated to this energy integral.

Since the $p$-harmonic map is a generalization of harmonic map, it is natural to speculate whether theorems for the 2-harmonic maps can be extended to the case of $p$-harmonic maps. That this is the case was shown in the context of the regularity theory established by Hardt, Kinderlehrer and Lin [17] as well as Luckhaus [22] in the eighties. More precisely, they obtained partial regularity results for minimizing weak $p$-harmonic maps between manifolds, extending previous results of Schoen and Uhlenbeck (29] and [12]) concerning the regularity of 2-harmonic maps.

If $p$ coincides with the dimension of the domain manifold, the $p$-energy is a conformal invariant. Such a $p$-harmonic map is called either a conformal $p$-harmonic map or an $m$-harmonic map, where $m$ denotes the dimension of the domain manifold. To date, not much is known on the $m$-harmonic maps for $m \geq 3$, although it is widely believed that $m$-harmonic maps enjoy properties similar to harmonic maps on surfaces due to the effect of conformal invariance (see e.g. [25], p. 357, [24], p. 169). To name just one example substantiating this belief, we mention the works of Strzelecki and Fuchs on Hölder continuity of weak $m$-harmonic maps from the $m$-ball to the $m$-sphere. This is an analogue of Hélein's regularity theorem in the context of harmonic maps (see [11, 27]). Bearing in mind this and other examples, the formal similarity between $m$-harmonic flow and harmonic flow on surfaces led us naturally to consider finite time blow-up of these heat flows.

Parallel to the developments on extending results of 2-harmonic maps to $p$ harmonic maps, there have been some works on the $p$-harmonic map heat flows (see e.g. 3], 20] and especially the literature cited in the latter paper). In the conformal case, Hungerbühler [20] proved the global existence of a partially regular weak solution of $m$-harmonic flow when both the domain and target are compact manifolds without boundary.

Historically, the development is as follows. In 1964, Eells and Sampson studied the heat flow associated to the 2-harmonic map and showed that for $C^{1}$ initial values, solutions to the heat flow equations exist at least locally in time. In case 
the target manifold is non-positively curved, they established the global existence of solutions to the 2-harmonic map heat flow. On the other hand, when the target manifold is positively curved, the loss of the compactness phenomenon occurs, as shown by Sachs and Uhlenbeck [28. Later, Struwe [30] gave a more quantitative result by establishing the global existence and uniqueness of partially regular weak solutions of harmonic flow on surfaces (a conformal case). More recently, Hungerbühler generalized Struwe's result to the higher-dimensional conformal case [20] as mentioned earlier.

In addition to the analogy with harmonic map heat flow on surfaces, our study of blow-up of conformal $p$-harmonic heat flow is motivated by the fact that the rotationally symmetric conformal $p$-harmonic maps are identical for all $p$ (see [4]), in the sense that they can be parametrized by the same family of functions. This family of functions was employed in the construction of comparison functions in the 2-harmonic flow by Chang, Ding and Ye to show finite time blow-up. Because of this, we were convinced that a comparison argument similar to that of Chang, Ding and Ye might be realizable in case the $p$-energy is a conformal invariant. Nevertheless, it turns out that several difficulties need to be overcome to generalize this scheme to other conformal cases.

Instead of dealing with semilinear uniformly parabolic equations in the 2-harmonic case, we need to treat quasilinear parabolic equations which are possibly degenerate. Owing to these, there are four major technical difficulties which we have to overcome. First, the arguments needed to show that rotationally symmetric initial data generate a rotationally symmetric solution is more delicate than the one used in Chang, Ding and Ye [2]. Second, to construct a lower solution, we have to rely on the rotationally symmetric $m$-harmonic maps and a carefully chosen perturbation term and the resulting asymptotic calculation is lengthy. This is the point where we are forced to restrict to the case $m=3$. Third, to satisfy parabolic degeneracy, we have to extend the blow-up results to cover weak solutions belonging to $C^{1}$, since there is no regularity bootstrap for such degenerate equations to conclude that a $C^{1}$ weak solution is $C^{2}$. Indeed, for the $m$-harmonic heat flow case, modulo finitely many points $t_{1}, t_{2}, \ldots, t_{k}$ in time, the gradient of the solutions is in $C^{\alpha}$. However, it is not known if the solution can be bootstraped to $C^{2}$ (see Theorem 10 in [20]).

Remark 1.1. Hungerbühler (see Remark on p. 628 in [20]) conjectured the finite time blow-up for any weak $m$-harmonic flow between two compact manifolds without boundary. He also obtained numerical evidence for his conjecture. Our result may be thought of as the first step towards Hungerbühler's conjecture. In general, one can ask what combinations of manifold geometry, initial data, and boundary conditions will lead to finite time blow-up.

Remark 1.2. A slightly modified version of the proof of Theorem 1.3 indicates a similar blow-up for some $n>p=3$. We conjecture that it may require only $n \geq p$.

1.2. Main result. We first introduce some notation to simplify the formulation in equations. Let $r=|x|$ and $\tilde{S} \equiv\left\{\varphi \in C^{1}[0,1]: \varphi(0)=0\right\}$. For any radial function $\varphi \in \tilde{S}$, we define a density function $\theta: \tilde{S} \rightarrow C[0,1]$ by

$$
\theta(\varphi) \equiv \varphi_{r}^{2}+(n-1) \sin ^{2} \varphi / r^{2} .
$$

We will suppress the dependence of $\theta$ when there is no ambiguity about its argument. Assume that 
(A1): $\varphi_{0}:[0,1] \rightarrow[0, \infty)$ is a $C^{2+\alpha}$ function of the radial distance $r$ with some $\alpha \in(0,1)$. Moreover, $\varphi_{0}(0)=0, \varphi_{0}^{\prime \prime}(0)=0, \varphi_{0}>0$ for $r \in(0,1], \varphi_{0}^{\prime}(0)>0$, and $\theta=\theta\left(\varphi_{0}\right) \neq 0$ in $[0,1]$.

If the initial condition has the form

$$
u_{0}(x)=\left(\frac{x}{r} \sin \varphi_{0}(r), \cos \varphi_{0}(r)\right),
$$

then $u_{0} \in C^{2+\alpha}\left(\overline{D^{3}}\right)$. This follows from (an analogue of) Lemma 2.1 in [1]:

Lemma 1.1. $u \equiv\left(\frac{x}{r} \sin \varphi(r), \cos \varphi(r)\right) \in C^{2+\alpha}\left(\overline{D^{3}}\right)$ if and only if $\varphi \in\{f \in$ $\left.C^{2+\alpha}[0,1]: f(0)=f_{r r}(0)=0\right\}$.

It will be shown that with the initial condition (1.3), the solution $u$ to equations (1.1) and (1.2) takes the same form: $u(x, t)=\left(\frac{x}{r} \sin \varphi, \cos \varphi\right)$, provided $\varphi$ is a radial function satisfying

$$
\begin{aligned}
\frac{\partial \varphi}{\partial t} & =\left(\theta^{\frac{p}{2}-1} \varphi_{r}\right)_{r}+\frac{n-1}{r} \theta^{\frac{p}{2}-1} \varphi_{r}-\frac{n-1}{r^{2}} \theta^{\frac{p}{2}-1} \sin \varphi \cos \varphi \\
& =\theta^{\frac{p}{2}-1}\left\{\varphi_{r r}+\frac{n-1}{r} \varphi_{r}+\left(\frac{p}{2}-1\right) \frac{\theta_{r}}{\theta} \varphi_{r}-\frac{n-1}{r^{2}} \sin \varphi \cos \varphi\right\},
\end{aligned}
$$

where $\theta=\theta(\varphi)$. The corresponding initial and boundary conditions then become

$$
\left\{\begin{array}{l}
\varphi(r, 0)=\varphi_{0}(r), \\
\varphi(0, t)=\varphi_{0}(0)=0, \varphi(1, t)=\varphi_{0}(1) \equiv b .
\end{array}\right.
$$

Using a comparison theorem, Chang, Ding, and Ye 2] proved a finite time blow-up of the spatial derivative $\varphi_{r}$ for the heat flow of harmonic maps from $D^{2}$ to $S^{2}$ under certain initial and boundary conditions. The objective of this paper is to generalize this result to the 3 -harmonic flow from $D^{3}$ to $S^{3}$. An overview of the technical difficulties involved is listed at the end of the section.

Our main theorems follow:

Theorem 1.1. Suppose $n=p=3$. Let the initial conditions $u_{0} \in C^{2+\alpha}\left(\overline{D^{3}}\right)$ and $\left|\nabla u_{0}\right| \neq 0$. Then for some $T>0$, there exists a unique solution in $C^{2+\alpha, 1+\alpha / 2}\left(\overline{D^{3}} \times\right.$ $[0, T])$ to the 3-harmonic map heat flow equations (1.1) and (1.2).

Theorem 1.2. Suppose $n=p=3$. Let $\varphi_{0}$ satisfy assumption (A1) and $u_{0}$ be rotationally symmetric as given by (1.3). Then the unique solution in Theorem 1.1] takes up the same symmetry: $u(x, t)=\left(\frac{x}{r} \sin \varphi, \cos \varphi\right)$, with $\varphi \in C^{2+\alpha, 1+\alpha / 2}([0,1] \times$ $[0, T])$ and satisfies equation (1.5) with initial and boundary conditions (1.6). This solution can be extended in $t$ as long as its $C^{2+\alpha, \frac{2+\alpha}{2}}$ norm is finite and its energy density $\theta$ does not vanish.

The smooth rotationally symmetric solution constructed in Theorem 1.2 also satisfies the governing equations in a weak (distribution) sense. We require our weak solution to be of $C^{1}$ in $[0,1] \times[0, T]$ (see Remark 3.1 for a less restrictive definition such that our theorem below still works). Its precise definition is given in section 3 .

Theorem 1.3. Let the same assumptions as in Theorem 1.2 hold. If $\left|\varphi_{0}(1)\right|>\pi$, then there is no global rotationally symmetric weak solution $u$ to (1.1). The uniform norm of $\varphi_{r}$ (hence that of $|\nabla u|$ ) is to blow up in finite time. 
The proof of Theorem [1.3 is based on the method of upper and lower solutions. The same method was used by Chang, Ding and Ye [2] to study the case of $p=n=$ 2. Their steady state solutions for (1.5) are $2 \cos ^{-1}\left(r / \sqrt{r^{2}+\lambda^{2}}\right)$ or $2 \tan ^{-1}(r / \lambda)$, for any $\lambda>0$. By adding a perturbation term, they arrive at their lower solution (or subsolution):

$$
v(r, t)=2 \tan ^{-1}\left(\frac{r}{\lambda(t)}\right)+2 \tan ^{-1}\left(\frac{r^{\gamma}}{\mu}\right) .
$$

Here $\mu$ is a large parameter, $\gamma \in(1,2)$, and $\lambda$ is a function of $t$, satisfying

$$
\frac{d \lambda}{d t}=-\delta \lambda^{\omega}
$$

with $\omega \equiv \gamma-1$. Hence $\lambda$ will decrease from some positive $\lambda_{0}$ to 0 in finite time, so that the spatial derivative of $v$ has to be blow-up in finite time.

We make two observations. First, the perturbation term $2 \tan ^{-1}\left(\frac{r^{\gamma}}{\mu}\right)$ is essentially $\epsilon r^{\gamma}$, with $\epsilon \equiv 2 / \mu$ being a small parameter. The latter term is more convenient when we calculate the asymptotic expansions for the subsolution in terms of $\epsilon, r$ and $t$.

The second observation is that $2 \tan ^{-1}(r / \lambda)$ is still a steady state solution when $p=n=3$. So we let

$$
v(r, t)=2 \tan ^{-1}\left(\frac{r}{\lambda(t)}\right)+\epsilon r^{\gamma},
$$

and go through a lengthy asymptotic expansion to establish $v$ as a subsolution. It is also clear from its proof that Theorem 1.3 can be generalized to 3 -harmonic heat flow from a 3-dimensional model space to $S^{n}$ (cf. [5]). Currently, we cannot find a subsolution that works for $p=n \geq 4$.

Remark 1.3. We note that for Chang, Ding and Ye's choice of subsolution, a lot of trigonometry is involved in proving the analogue of our Lemma 4.1 Our choice of subsolution is more instructive and easier to estimate.

The organization of this paper is as follows. In the next section we prove Theorems 1.1 and 1.2. Section 3 is devoted to the maximum principle for weak upper and lower solutions. Then in section 4 , we prove that the function $v$ defined above is a strict subsolution and complete the blow-up argument. Finally, a key lemma used in section 4 will be proved in section 5 .

\section{LOCAL EXISTENCE AND UNIQUENESS OF SOLUTIONS}

In this section we fix $n=p=3$. In contrast to the 2-harmonic heat flow, the terms involving the highest spatial derivatives in the 3-harmonic case are nonlinear. This may cause failure in adapting some workable schemes used to establish the local existence and uniqueness for the 2-harmonic flow to the 3-harmonic flow.

We will adopt a strategy which applies equally well in our case (p. 122, [16]). In [16], the linearized version of the governing equations was studied. With appropriate a priori bounds for the linearized equations, Hamilton proved the local existence and uniqueness by making use of the inverse function theorem.

To duplicate this argument, there are two sources of technical difficulty that we must overcome: the linearized parabolic equations are strongly coupled and may be degenerate. 
First we focus on the existence of smooth solutions. Let $0<\alpha<1$. Initial data $u_{0}$ is assumed to be in $C^{2+\alpha}$ and nondegenerate in the sense that $\left|\nabla u_{0}\right| \neq 0$ in $\overline{D^{3}}$. Such initial conditions will avoid the degeneracy of the corresponding linearized parabolic system.

Let $Q_{1}=D^{3} \times(0,1)$ and $1 \leq p<\infty$. With reference to [23, p. 5], we define the Sobolev spaces $L^{p}$ and $W_{p}^{2,1}$ on $Q_{1}$ respectively as follows:

$$
\begin{aligned}
\|\varphi\|_{p} & \equiv\left(\int_{Q_{1}}|\varphi(x, t)|^{p} d x d t\right)^{1 / p} \\
\|\varphi\|_{W_{p}^{2,1}} & \equiv\|\varphi\|_{p}+\left\|\varphi_{x}\right\|_{p}+\left\|\varphi_{x x}\right\|_{p}+\left\|\varphi_{t}\right\|_{p} .
\end{aligned}
$$

By the Sobolev type imbedding theorem (p. 80, 23]), with sufficiently large $p$, $W_{p}^{2,1}\left(Q_{1}\right) \subset C^{1+\alpha,(1+\alpha) / 2}\left(\overline{Q_{1}}\right)$. We will fix such a $p$ from now on. A function $\varphi \in W_{0, p}^{2,1}\left(Q_{1}\right)$ if it is in $W_{p}^{2,1}\left(Q_{1}\right)$ and $\varphi=0$ at the parabolic boundary of $Q_{1}$, i.e., at both $t=0$ and $\partial D^{3}$.

Define $F:\left(W_{p}^{2,1}\left(Q_{1}\right)\right)^{4} \rightarrow\left(L_{p}\left(Q_{1}\right)\right)^{4}$ such that if $u \in\left(W_{p}^{2,1}\left(Q_{1}\right)\right)^{4}$,

$$
F(u) \equiv \nabla \cdot(|\nabla u| \nabla u)+|\nabla u|^{3} u-u_{t} .
$$

Next, let $G:\left(W_{0, p}^{2,1}\left(Q_{1}\right)\right)^{4} \rightarrow\left(L_{p}\left(Q_{1}\right)\right)^{4}$ such that if $v \in\left(W_{0, p}^{2,1}\left(Q_{1}\right)\right)^{4}$, then $G(v) \equiv$ $F\left(u_{0}+v\right)$. We note that $u=u_{0}+v$ is in $S$, where

$$
S \equiv\left\{u \in\left(W_{p}^{2,1}\left(Q_{1}\right)\right)^{4}: u=u_{0} \text { for } x \in \partial D^{3}, \text { and } u(x, 0)=u_{0}\right\} .
$$

It can be checked that both $F$ and $G$ are continuously Frechet differentiable. In fact, calculations show that for any $w \in\left(W_{0, p}^{2,1}\left(Q_{1}\right)\right)^{4}$,

$$
\begin{aligned}
& G^{\prime}(0) w=F^{\prime}\left(u_{0}\right) w=\nabla \cdot\left(\left|\nabla u_{0}\right| \nabla w\right)+\nabla \cdot\left[\frac{1}{\left|\nabla u_{0}\right|}\left(\nabla u_{0} \cdot \nabla w\right) \nabla u_{0}\right] \\
& \quad+\left|\nabla u_{0}\right|^{3} w+3 u_{0}\left|\nabla u_{0}\right| \nabla u_{0} \cdot \nabla w-w_{t} \\
& \equiv \sum_{\beta, i, j} A_{i j}^{\gamma \beta} D_{i j} w^{\beta}+\sum_{\beta, i} B_{i}^{\gamma \beta} D_{i} w^{\beta}+C w^{\gamma}-w_{t}^{\gamma},
\end{aligned}
$$

which is a strongly coupled system. Let $\delta_{i j}$ be 1 if $i=j$ and 0 if $i \neq j$, for $i=1,2,3$, and $j=1,2,3$. The same is true for $\delta^{\gamma \beta}$ for $\gamma=1, \ldots, 4$, and $\beta=1, \ldots, 4$. It can be checked that $C$ is in $L^{\infty}, B_{i}^{\gamma \beta}$ are in $L^{p}$, and $A_{i j}^{\gamma \beta}=\frac{1}{\left|\nabla u_{0}\right|}\left\{\left|\nabla u_{0}\right|^{2} \delta_{i j} \delta^{\gamma \beta}+\right.$ $\left.D_{i} u_{0}^{\gamma} D_{j} u_{0}^{\beta}\right\}$, which are continuous in $\overline{D^{3}}$. It is clear that $A_{i j}^{\gamma \beta}=A_{j i}^{\beta \gamma}$. Moreover, for any $\xi=\left(\xi_{i}^{\gamma}\right) \in \mathbf{R}^{12}$ with $|\xi|=1$, there exists a $\lambda>0$ such that

$$
\sum_{\beta, \gamma, i, j} A_{i j}^{\gamma \beta} \xi_{i}^{\gamma} \xi_{j}^{\beta}=\frac{1}{\left|\nabla u_{0}\right|}\left\{\left|\nabla u_{0}\right|^{2}+\left(\sum_{\beta, j} \xi_{j}^{\beta} D_{j} u_{0}^{\beta}\right)^{2}\right\} \geq\left|\nabla u_{0}\right| \geq \lambda>0 .
$$

Hence the linearized system satisfies the (strong) Legendre condition, and is uniformly parabolic.

With all the hypotheses of Theorem 2 of [26] being satisfied, given any $f \in$ $\left(L_{p}\left(Q_{1}\right)\right)^{4}$, there exists a unique $w \in\left(W_{0, p}^{2,1}\left(Q_{1}\right)\right)^{4}$ satisfying $G^{\prime}(0) w=f$, with a constant $C>0$ such that

$$
\|w\|_{W_{p}^{2,1}} \leq C\|f\|_{p}
$$

Such an estimate allows us to employ the inverse function theorem below.

Note. In Theorem 2 of 26, they derive an a priori bound only. However, the method of continuity (p. 75, 13]) will give us the existence of a solution once we have the a priori bounds. Furthermore, they require that the coefficients $B_{i}^{\gamma \beta}$ in (2.1) be in $L^{\infty}$. For our choice of large $p$, the same proof shows that $B_{i}^{\gamma \beta}$ being in 
$L^{p}$ suffices. For a scalar equation, the result for such coefficient $B$ can be found on p. $341,[23]$.

Now $G(0)=F\left(u_{0}\right) \in\left(L_{p}\left(Q_{1}\right)\right)^{4}$. Let $\delta>0$. Define a function $f$ such that $f=0$ for $0<t<\delta$ and $f=G(0)$ for $\delta<t<1$. Hence $f$ is in a small neighborhood of $G(0)$ in $\left(L_{p}\left(Q_{1}\right)\right)^{4}$ norm. Using the inverse function theorem, provided $\delta$ is small enough, there exists a unique $v \in\left(W_{0, p}^{2,1}\left(Q_{1}\right)\right)^{4}$ such that $G(v)=f$. In other words, for $0<t<\delta, F\left(u_{0}+v\right)=0$. Since $u=u_{0}+v$ is in $S$, the local existence and uniqueness of a solution in $S$ has been established.

We next bootstrap this solution to higher regularity. Rewrite the equation (1.1) as

$$
u_{t}^{\gamma}=\sum_{\beta, i, j} \tilde{A}_{i j}^{\gamma \beta} D_{i j} u^{\beta}+|\nabla u|^{3} u^{\gamma}
$$

with $\tilde{A}_{i j}^{\gamma \beta} \equiv \frac{1}{|\nabla u|}\left\{|\nabla u|^{2} \delta_{i j} \delta^{\gamma \beta}+D_{i} u^{\gamma} D_{j} u^{\beta}\right\}$. By reducing $\delta$ if necessary, we can assume $|\nabla u| \neq 0$ for $0 \leq t \leq \delta$. Since $u \in\left(W_{p}^{2,1}\left(Q_{1}\right)\right)^{4}$, the coefficients $\tilde{A}$ and the source term $|\nabla u|^{3} u$ are Hölder continuous. Hence Schauder's estimate for a strongly coupled system (Theorem 1 in [26]) gives each component $u^{\gamma}, \gamma=1, \ldots, 4$, as being in $C^{2+\alpha,(2+\alpha) / 2}\left(\overline{D^{3}} \times[0, \delta]\right)$. This completes the proof of Theorem 1.1

Next we are going to show that "rotational symmetry" is preserved for initial data (1.3) under time evolution. That is, $u(x, t)=\left(\frac{x}{r} \sin \varphi, \cos \varphi\right)$ for some radial function $\varphi$ satisfying (1.5). The proof is in the same spirit as the treatment of 2-harmonic maps [1], though the calculation in our case is more involved.

Let $(r, \beta, \gamma)$ be the usual spherical coordinates in $D^{3}$, with $0 \leq r \leq 1,0 \leq \beta \leq \pi$, and $0 \leq \gamma \leq 2 \pi$ (see (2.66) below). Since $u: D^{3} \rightarrow S^{3}$, we can let

$$
u=(\sin a \sin b \cos c, \sin a \sin b \sin c, \sin a \cos b, \cos a)^{T},
$$

with $a, b, c$ being functions of $r, \beta, \gamma$, and $t$. We have $0 \leq a \leq \pi, 0 \leq b \leq \pi$, and $0 \leq c \leq 2 \pi$.

As $u$ moves continuously on $S^{3}$, its spherical coordinates will change continuously. Hence $a, b$, and $c$ are continuous functions of $u_{1}$ to $u_{4}$. Since each $u_{i}$ is a continuous function of $x_{1}, x_{2}, x_{3}$, and $t$; thus,

$$
a=a\left(x_{1}, x_{2}, x_{3}, t\right), \quad b=b\left(x_{1}, x_{2}, x_{3}, t\right), \quad c=c\left(x_{1}, x_{2}, x_{3}, t\right)
$$

are in $C\left(\overline{D^{3}} \times[0, \delta]\right)$. Next we compute the $4 \times 3$ Jacobian matrix $J$ for the transformation (2.2):

$$
\begin{aligned}
J & \equiv\left(\begin{array}{ccc}
\frac{\partial u_{1}}{\partial a} & \cdot & \frac{\partial u_{1}}{\partial c} \\
\cdot \cdot & \cdot & \cdot \\
\frac{\partial u_{4}}{\partial a} & \cdot & \frac{\partial u_{4}}{\partial c}
\end{array}\right) \\
& =\left(\begin{array}{ccc}
\cos a \sin b \cos c & \sin a \cos b \cos c & -\sin a \sin b \sin c \\
\cos a \sin b \sin c & \sin a \cos b \sin c & \sin a \sin b \cos c \\
\cos a \cos b & -\sin a \sin b & 0 \\
-\sin a & 0 & 0
\end{array}\right) .
\end{aligned}
$$

If $\sin a \sin b \neq 0$, then $\operatorname{rank}(J)=3$. Under such a condition, the inverse function theorem gives $a=a\left(u_{i}, u_{j}, u_{k}\right)$ being $C^{\infty}$ for some distinct $i, j, k$ in the set $\{1,2,3,4\}$. With each $u_{i}, i=1, \ldots, 4$, being in $C^{2,1}\left(\overline{D^{3}} \times[0, \delta]\right), a=a\left(x_{1}, x_{2}, x_{3}, t\right)$ 
is $C^{2,1}$ at those $(\mathbf{x}, t)$ such that $\sin a \sin b \neq 0$. Similarly, $b=b(\mathbf{x}, t)$ and $c=c(\mathbf{x}, t)$ are in $C^{2,1}$ under the same condition. With abuse of notation, we will use the same symbols $a, b$ and $c$, when they are functions of $(\mathbf{x}, t)$ and as functions of the spherical coordinates $(r, \beta, \gamma, t)$.

We now identify $D^{3}$ with $D \equiv\left\{x \in \mathbf{R}^{4}: x=\left(x_{1}, x_{2}, x_{3}, 0\right), x_{1}^{2}+x_{2}^{2}+x_{3}^{2}<1\right\}$, and regard $u: D \rightarrow S^{3}$. Let $T_{\theta}: D \rightarrow D$ be any rotation through an angle $\theta$ while keeping the $x_{4}$ axis fixed. Because $T_{\theta}^{-1} u\left(T_{\theta} x, t\right)$ satisfies (1.1), and the initial and the boundary conditions, we have

$$
T_{\theta}^{-1} \circ u(\cdot, t) \circ T_{\theta}=u(\cdot, t)
$$

due to the uniqueness of solutions to equations (1.1) and (1.2).

There are two independent angles of rotation for $T_{\theta}$. Hence for any $\psi$ and $\phi$, (2.4) leads to

$$
\begin{array}{ll}
a(r, \beta+\psi, \gamma, t)=a(r, \beta, \gamma, t), & a(r, \beta, \gamma+\phi, t)=a(r, \beta, \gamma, t), \\
b(r, \beta+\psi, \gamma, t)=\psi+b(r, \beta, \gamma, t), & b(r, \beta, \gamma+\phi, t)=b(r, \beta, \gamma, t), \\
c(r, \beta+\phi, \gamma, t)=c(r, \beta, \gamma, t), & c(r, \beta, \gamma+\phi, t)=\phi+c(r, \beta, \gamma, t),
\end{array}
$$

provided $r \neq 0$. Thus $a=a(r, t), b=b(r, \beta, t)=\beta+b_{1}(r, t)$, and $c=c(r, \gamma, t)=$ $\gamma+c_{1}(r, t)$ for some functions $b_{1}$ and $c_{1}$. Consequently, if $r \neq 0$,

$$
\begin{aligned}
u(r, \beta, \gamma, t)= & \left(\sin a \sin \left(\beta+b_{1}\right) \cos \left(\gamma+c_{1}\right),\right. \\
& \left.\sin a \sin \left(\beta+b_{1}\right) \sin \left(\gamma+c_{1}\right), \sin a \cos \left(\beta+b_{1}\right), \cos a\right)^{\mathrm{T}} .
\end{aligned}
$$

Our aim of showing the spherical symmetric solution as time evolves amounts to proving $b_{1}=c_{1}=0$. It is noted that in obtaining the symmetry condition (2.5), smoothness of $a, b$, and $c$ are not needed.

Recall that

$$
x_{1}=r \sin \beta \cos \gamma, \quad x_{2}=r \sin \beta \sin \gamma, \quad x_{3}=r \cos \beta .
$$

We compute the $3 \times 3$ Jacobian matrix $\tilde{J}$ of $x_{i}$ with respect to $r, \beta$, and $\gamma$. If $r^{2} \sin \beta \neq 0, \operatorname{rank}(\tilde{J})=3$, the inverse function theorem then implies that $r, \beta$ and $\gamma$ are $C^{\infty}$ functions of $x_{1}, x_{2}$, and $x_{3}$.

Set $\beta=\pi / 2$ and $\gamma=0$. Then $b_{1}(r, t)=b(r, \pi / 2,0, t)-\pi / 2$ is a $C^{2,1}$ function of $(r, t)$, if $\sin a \cos b_{1} \neq 0$ and $r \neq 0$. Moreover, because of (2.3), $\lim _{r \rightarrow 0} b_{1}(r, t)$ exists. Hence $b_{1}$ is continuous in $(r, t)$ on $[0,1] \times[0, \delta]$. Since $b_{1}=0$ at $t=0$, by shrinking the time $\delta$ if necessary, we can assume that $\cos b_{1} \neq 0$. Hence we conclude that $b_{1}(r, t)$ is in $C([0,1] \times[0, \delta])$ and is in $C^{2,1}$ whenever $\sin a \neq 0$ and $r \neq 0$. The same conditions for these two different kinds of smoothness apply to $a$ and $c_{1}$.

We now claim that $\sin a=0$ at $r=0$. This is because $u_{3}=\sin a \cos b$ is continuous at the origin, $\lim _{r \rightarrow 0} \sin a \cos \left(b_{1}+\beta\right)$ exists no matter what $\beta$ is. This is possible only when $\sin a(0, t)=0$ for all time $t$.

Let $v \equiv \sin a \sin b_{1}$. For those $(\mathbf{x}, t)$ such that $\sin a \neq 0$ and $r \neq 0$, the governing equation on $v$ is (see Appendix 1) in the form of $v_{t}=A v_{r r}+B v_{r}+C v$ with coefficients $A=A(r, t), B=B(r, t)$, and $C=C(r, t)$. Moreover, $A$ is uniformly parabolic and $C$ is bounded from above. With boundary condition $b_{1}(1, t)=0$ and initial condition $b_{1}(r, 0)=0$, we have $v(1, t)=0$ and $v(r, 0)=0$. Since $\sin a=0$ at $r=0$, we also have the boundary condition $v(0, t)=0$.

Assume $b_{1} \neq 0$ for some $\left(r_{0}, t_{0}\right)$ at which $\sin a \neq 0$. We will show that this is impossible. With $v$ in $C([0,1] \times[0, \delta])$, and $v=0$ whenever $\sin a=0$, we can apply 
the classical maximum principle to conclude that $v=0$ in the whole domain. This requires $b_{1}\left(r_{0}, t_{0}\right)=0$ whenever $\sin a \neq 0$, which is a contradiction.

When $\sin a=0$, we can assign any value to $b_{1}$ without changing the value of $u$. Hence we have proved $b_{1}=0$ in $[0,1] \times[0, \delta]$.

Let $\tilde{w}=\sin a \sin c_{1}$. Similarly, we derive an equation on $\tilde{w}$ in Appendix 2 and conclude using the same argument that $c_{1}=0$. Writing $a$ as $\varphi$, then $u$ as given by (2.2) simplifies to $u=\left(\frac{x}{r} \sin \varphi, \cos \varphi\right)$. The governing equation (1.5) on $\varphi$ corresponds to the fourth equation in (1.1) with $u_{4}=\cos \varphi$. Therefore, we have shown that rotationally symmetric initial data leads to a rotationally symmetric solution.

\section{MAXimum PRINCIPLE FOR WEAK SOlUtions}

In order to study the finite time blow-up, we need a comparison principle for the method of upper and lower solutions. We will establish such a principle for the case $n=p=3$.

When the initial data is smooth with the corresponding $\theta$ being nonzero, we have shown that there is a smooth $C^{2,1}([0,1] \times[0, T))$ solution to (1.6), provided $T>0$ is sufficiently small. As time evolves, $\theta$ may become zero, and the parabolic equation is degenerate. As a matter of fact, this requires an interpretion of the term involving $1 / \sqrt{\theta}$ in equation (1.6). On the other hand, the divergence form (1.4) can be employed to define a weak (distribution) solution, even when there is a loss of strict parabolicity. From now on, we let $Q_{T} \equiv(0,1) \times(0, T)$.

By a weak upper solution $u$ of equation (1.4), we mean a function in $C^{1}\left(\overline{Q_{T}}\right)$, satisfying

$$
\left(\sqrt{\theta} u_{r}\right)_{r}+\frac{2}{r} \sqrt{\theta} u_{r}-\frac{2}{r^{2}} \sqrt{\theta} \sin u \cos u-u_{t} \leq 0
$$

in a weak sense. In other words, for any positive test function $\psi \in C^{\infty}\left(\overline{Q_{T}}\right)$ having compact support near $x=0$ and $x=1$ for all time, we have

$$
\begin{aligned}
-\left.\int_{0}^{1} u \psi d r\right|_{t=0} ^{T_{0}}+\int_{0}^{T_{0}} \int_{0}^{1}\{ & -\sqrt{\theta} u_{r} \psi_{r}+\frac{2}{r} \psi \sqrt{\theta} u_{r} \\
& \left.-\frac{2}{r^{2}} \psi \sqrt{\theta} \sin u \cos u+u \psi_{t}\right\} d r d t \leq 0 .
\end{aligned}
$$

A weak lower solution $v$ is defined in the same manner with a sign change in the above inequality. A weak solution is both a weak upper and a weak lower solution. It is clear that a smooth solution is a weak solution.

Remark 3.1. We can relax the smoothness requirement on the upper solution $u$. Actually we need only both $u$ and $u_{x}$ to be continuous in $\overline{Q_{T}}$. There is no requirement on the existence of $u_{t}$. An additional standard modification will then be required in our proof for the comparison principle. A similar remark applies to the lower solution. We will give further comment on such issues during the proof.

Lemma 3.1. Let $u$ and $v$ be the weak upper and weak lower solutions to equation (1.4), respectively. Suppose $u$ and $v$ satisfy the boundary conditions $u(0, t)=$ $v(0, t)=0, v(1, t) \leq u(1, t)$ for any $t \in[0, T]$, and the initial conditions $v(r, 0) \leq$ $u(r, 0)$ for any $r \in[0,1]$. For some $\delta_{1}>0$, if $v_{r}(0, t) \geq \delta_{1}$ for any $t \in[0, T]$, then $v \leq u$ in $\overline{Q_{T}}$. 
Proof. Define $w=w(r, t, \xi) \equiv v+\xi(u-v)$, with $0 \leq \xi \leq 1$, and $\Theta(w) \equiv w_{r}^{2}+$ $2 \sin ^{2} w / r^{2}$. Similarly, $\Theta(u) \equiv u_{r}^{2}+2 \sin ^{2} u / r^{2}$, and $\Theta(v) \equiv v_{r}^{2}+2 \sin ^{2} v / r^{2}$. Take any $\epsilon>0$; then for any fixed $r>0$ and $t$,

$$
\begin{gathered}
u_{r} \sqrt{\Theta(u)+\epsilon}-v_{r} \sqrt{\Theta(v)+\epsilon}=\int_{0}^{1}\left\{\frac{w_{r}^{2}}{\sqrt{\Theta(w)+\epsilon}}\left(u_{r}-v_{r}\right)+\sqrt{\Theta(w)+\epsilon}\left(u_{r}-v_{r}\right)\right. \\
\left.+\frac{2 w_{r} \sin w \cos w}{r^{2} \sqrt{\Theta(w)+\epsilon}}(u-v)\right\} d \xi
\end{gathered}
$$

Since $\left\|w_{r} / \sqrt{\Theta(w)+\epsilon}\right\|_{\infty} \leq 1,\|\sqrt{2} \sin w /(r \sqrt{\Theta(w)+\epsilon})\|_{\infty} \leq 1$, together with the assumption that $u, u_{r}, v$, and $v_{r}$ are continuous in $\overline{Q_{T}}$, we can take the limit as $\epsilon \rightarrow 0$ using the dominated convergence theorem to obtain

$$
\begin{gathered}
u_{r} \sqrt{\Theta(u)}-v_{r} \sqrt{\Theta(v)} \\
=\int_{0}^{1}\left\{\frac{w_{r}^{2}}{\sqrt{\Theta(w)}}\left(u_{r}-v_{r}\right)+\sqrt{\Theta(w)}\left(u_{r}-v_{r}\right)\right. \\
\left.\quad+\frac{2 w_{r} \sin w \cos w}{r^{2} \sqrt{\Theta(w)}}(u-v)\right\} d \xi \\
\equiv \int_{0}^{1}\{I\} d \xi,
\end{gathered}
$$

where $I$ represents all the terms inside the parenthesis. Similarly

$$
\begin{aligned}
& \sin (2 u) \sqrt{\Theta(u)}-\sin (2 v) \sqrt{\Theta(v)} \\
& =\int_{0}^{1}\left\{\sin (2 w)\left[\frac{w_{r}}{\sqrt{\Theta(w)}}\left(u_{r}-v_{r}\right)+\frac{2 \sin w \cos w}{r^{2} \sqrt{\Theta(w)}}(u-v)\right]\right. \\
& \quad+2 \cos (2 w) \sqrt{\Theta(w)}(u-v)\} d r \\
& \equiv \int_{0}^{1}\{I I I\} d \xi .
\end{aligned}
$$

We note that the first two terms inside the double integral of (3.1) will be treated by studying quantity I, and the third term by studying quantity III.

An analogous inequality such as (3.1) can be constructed for the lower solution $v$ with the inequality sign reversed. On subtracting it from (3.1), we obtain

$$
\begin{aligned}
\int_{Q_{T}} d r d t & \int_{0}^{1}\left\{-I \frac{\partial \psi}{\partial r}+\frac{2 I}{r} \psi-\frac{I I I}{r^{2}} \psi+(u-v) \frac{\partial \psi}{\partial t}\right\} d \xi \\
& -\left.\int_{0}^{1}(u-v) \psi d r\right|_{t=0} ^{T} \leq 0 .
\end{aligned}
$$

Let $\beta>0$ be a large number, whose magnitude will be determined later. Define $\tilde{u} \equiv u e^{-\beta t}, \tilde{v} \equiv v e^{-\beta t}$,

$$
\tilde{I} \equiv \frac{w_{r}^{2}}{\sqrt{\Theta(w)}}\left(\tilde{u}_{r}-\tilde{v}_{r}\right)+\sqrt{\Theta(w)}\left(\tilde{u}_{r}-\tilde{v}_{r}\right)+\frac{2 w_{r} \sin w \cos w}{r^{2} \sqrt{\Theta(w)}}(\tilde{u}-\tilde{v}),
$$

and a term $I \tilde{I} I$ by a similar definition. We note that in both $\tilde{I}$ and $I \tilde{I} I$, the terms involving $w$ and $\Theta$ are still constructed from $u$ and $v$, not $\tilde{u}$ and $\tilde{v}$. 
Observe that $\psi$ is a test function if and only if $\psi e^{\beta t}$ is a test function. Thus from (3.4), we can derive, for any test function $\psi$,

$$
\begin{aligned}
\int_{Q_{T}} d r d t \int_{0}^{1}\{ & -\tilde{I} \frac{\partial \psi}{\partial r}+\frac{2 \tilde{I}}{r} \psi-\frac{I \tilde{I} I}{r^{2}} \psi+(\tilde{u}-\tilde{v}) \frac{\partial \psi}{\partial t} \\
& -\beta(\tilde{u}-\tilde{v}) \psi\} d \xi-\left.\int_{0}^{1}(\tilde{u}-\tilde{v}) \psi d r\right|_{t=0} ^{T} \leq 0 .
\end{aligned}
$$

The extra term containing $\beta$ will help. (This is similar to the standard trick in making the coefficient $c$ in a parabolic equation to be negative, provided it is bounded above, so that one can apply the maximum principle.) Assume $\tilde{v}-\tilde{u} \geq \delta_{0}$ for some $\delta_{0}>0$ somewhere in $\overline{Q_{T}}$, we will show that there is a contradiction, leading us to conclude $v \leq u$. For any $\delta \leq \delta_{0} / 2$, take $\psi \equiv(\tilde{v}-\tilde{u}-\delta)^{+} \geq 0$ on $\overline{Q_{T}}$. Since $u$ and $v$ are $C^{1}$ functions, $\psi$ is therefore Lipschitz and has compact support due to the boundary conditions of $u$ and $v$. Although it is not smooth, we can still use this $\psi$ as our test function by a standard approximation argument. We further note that $\psi=0$ at $t=0$ due to the initial conditions of $u$ and $v$.

Define $Q_{\delta}^{+} \equiv\left\{(x, t) \in Q_{T}: \tilde{v} \geq \tilde{u}+\delta\right\}$. Then equation 3.5 gives

$$
\begin{aligned}
& \int_{Q_{\delta}^{+}} d r d t \int_{0}^{1}\{- \tilde{I} \frac{\partial}{\partial r}(\tilde{v}-\tilde{u})+\frac{2 \tilde{I}}{r}(\tilde{v}-\tilde{u}-\delta)-\frac{I \tilde{I} I}{r^{2}}(\tilde{v}-\tilde{u}-\delta) \\
&\left.+(\tilde{u}-\tilde{v}) \frac{\partial}{\partial t}(\tilde{v}-\tilde{u})-\beta(\tilde{u}-\tilde{v})(\tilde{v}-\tilde{u}-\delta)\right\} d \xi \\
&-\left.\int_{0}^{1}(\tilde{u}-\tilde{v})(\tilde{v}-\tilde{u}-\delta)^{+} d r\right|_{t=T_{0}} \leq 0 .
\end{aligned}
$$

Now we will push $\delta$ to 0 using the dominated convergence theorem; this allows us to replace $Q_{\delta}^{+}$by $Q_{T}^{+} \equiv\left\{(x, t) \in Q_{T}: \tilde{v} \geq \tilde{u}\right\}$ in 3.6 with all the $\delta$ being set to zero. We will analyse two of the terms in the equation

$$
\begin{aligned}
\int_{Q_{T}^{+}} & d r d t \int_{0}^{1}(\tilde{u}-\tilde{v}) \frac{\partial}{\partial t}(\tilde{v}-\tilde{u}) d \xi-\left.\int_{0}^{1}(\tilde{u}-\tilde{v})(\tilde{v}-\tilde{u})^{+} d r\right|_{t=T_{0}} \\
& =-\int_{Q_{T}} d r d t \frac{\partial}{\partial t}\left[\left((\tilde{v}-\tilde{u})^{+}\right)^{2} / 2\right]+\left.\int_{0}^{1}\left((\tilde{v}-\tilde{u})^{+}\right)^{2} d r\right|_{t=T} \\
& =\left.\frac{1}{2} \int_{0}^{1}\left((\tilde{v}-\tilde{u})^{+}\right)^{2} d r\right|_{t=T}
\end{aligned}
$$

Hence (3.6) becomes

$$
\begin{gathered}
\int_{Q_{T}^{+}} d r d t \int_{0}^{1}\left\{-\tilde{I} \frac{\partial}{\partial r}(\tilde{v}-\tilde{u})+\frac{2 \tilde{I}}{r}(\tilde{v}-\tilde{u})-\frac{I \tilde{I} I}{r^{2}}(\tilde{v}-\tilde{u})+\beta(\tilde{v}-\tilde{u})^{2}\right\} d \xi \\
+\left.\frac{1}{2} \int_{0}^{1}\left((\tilde{v}-\tilde{u})^{+}\right)^{2} d r\right|_{t=T_{0}} \leq 0 .
\end{gathered}
$$

If we prove the left-hand side in the above inequality is positive unless $\left|Q_{T}^{+}\right|$is zero, then we can conclude that $\tilde{v} \geq \tilde{u}$ in $\overline{Q_{T}}$. This violates $\tilde{v}-\tilde{u} \geq \delta_{0}$ somewhere. The proof of the lemma will thus be complete.

Note. We remark that the above calculations require $u_{t}$ and $v_{t}$ to be continuous. However, following the same calculations as on pp. 141 and 142 in [23], we can get the same results without assumption on the time derivatives. See Remark 3.1. 
We now put $\tilde{I}$ and $I \tilde{I} I$ into the above inequality. For example,

$$
-\tilde{I} \frac{\partial}{\partial r}(\tilde{v}-\tilde{u})=\left(\sqrt{\Theta(w)}+\frac{w_{r}^{2}}{\sqrt{\Theta(w)}}\right)\left(\tilde{v}_{r}-\tilde{u}_{r}\right)^{2}+\frac{w_{r} \sin (2 w)}{r^{2} \sqrt{\Theta(w)}}(\tilde{v}-\tilde{u})\left(\tilde{v}_{r}-\tilde{u}_{r}\right) .
$$

We then have

$$
\begin{aligned}
\int_{Q_{T}^{+}} d r d t \int_{0}^{1}\{ & \left(\sqrt{\Theta(w)}+\frac{w_{r}^{2}}{\sqrt{\Theta(w)}}\right)\left(\tilde{v}_{r}-\tilde{u}_{r}\right)^{2}+\frac{2 w_{r} \sin (2 w)}{r^{2} \sqrt{\Theta(w)}}(\tilde{v}-\tilde{u})\left(\tilde{v}_{r}-\tilde{u}_{r}\right) \\
& -\frac{2}{r}\left(\sqrt{\Theta(w)}+\frac{w_{r}^{2}}{\sqrt{\Theta(w)}}\right)(\tilde{v}-\tilde{u})\left(\tilde{v}_{r}-\tilde{u}_{r}\right) \\
& -\frac{2 w_{r} \sin (2 w)}{r^{3} \sqrt{\Theta}}(\tilde{v}-\tilde{u})^{2}+\frac{\sin ^{2}(2 w)}{r^{4} \sqrt{\Theta(w)}}(\tilde{v}-\tilde{u})^{2} \\
& \left.+\frac{2 \sqrt{\Theta(w)} \cos (2 w)}{r^{2}}(\tilde{v}-\tilde{u})^{2}+\beta(\tilde{v}-\tilde{u})^{2}\right\} d \xi \\
& +\left.\frac{1}{2} \int_{0}^{1}\left((\tilde{v}-\tilde{u})^{+}\right)^{2} d r\right|_{t=T_{0}} \leq 0
\end{aligned}
$$

Now denote the terms inside the parenthesis of the double integral by $I V$. Since the single integral term in (3.7) is always nonnegative, it suffices to show the double integral is positive unless $\left|Q_{T}^{+}\right|=0$.

By assumption, we know $v_{r}(0, t) \geq \delta_{1}>0$ for all $t \in[0, T]$. There are two cases to consider.

(i) First case: $u_{r}(0, t) \geq \delta_{1} / 2$ for all $t \in[0, T]$.

(ii) Second case: $u_{r}\left(0, T_{1}\right)=\delta_{1} / 2$ for some $T_{1}>0$. Without loss of generality, we assume $T_{1}$ to be the first time that such equality occurs. We will show that this case never exists.

(i) First case.

For any $t \in\left[0, T_{0}\right]$, any $\xi \in[0,1], w_{r}(0, t, \xi)=\xi u_{r}(0, t)+(1-\xi) v_{r}(0, t) \geq \delta_{1} / 2$. Let $r_{0}>0$ be small (whose magnitude will be determined later). With $u, v$, and their spatial derivatives being uniformly continuous in $\overline{Q_{T}}, w_{r}(r, t, \xi) \geq \delta_{1} / 4$ for any $r \leq r_{0}$. Moreover,

$$
\left|\frac{\sin (2 w)}{r}-2 w_{r}\right| \leq\left|\frac{\sin (2 w)-2 w}{r}\right|+\left|\frac{2 w}{r}-2 w_{r}\right| \leq|o(1)|,
$$

where $o(1) \rightarrow 0$ uniformly in $\xi \in[0,1], t \in\left[0, T_{0}\right]$, and $r \in\left[0, r_{0}\right]$ as $r_{0} \rightarrow 0$. Similarly,

$$
\left|\frac{\tilde{v}-\tilde{u}}{r}-\left(\tilde{v}_{r}-\tilde{u}_{r}\right)\right|=e^{-\beta t}\left|\frac{v-u}{r}-\left(v_{r}-u_{r}\right)\right|=o(1) e^{-\beta t},
$$

where $o(1)$ is independent of the magnitude of $\beta$.

We will use such information to manipulate the terms in $\{I V\}$. Define

$$
\begin{aligned}
(I V)_{a} & \equiv \sqrt{\Theta(w)}\left\{\left(\tilde{v}_{r}-\tilde{u}_{r}\right)^{2}-\frac{2}{r}(\tilde{v}-\tilde{u})\left(\tilde{v}_{r}-\tilde{u}_{r}\right)+\frac{2 \cos (2 w)}{r^{2}}(\tilde{v}-\tilde{u})^{2}\right\} \\
& =\sqrt{\Theta(w)}\left\{\left[\left(\tilde{v}_{r}-\tilde{u}_{r}\right)-\left(\frac{\tilde{v}-\tilde{u}}{r}\right)\right]^{2}+(2 \cos (2 w)-1)\left(\frac{\tilde{v}-\tilde{u}}{r}\right)^{2}\right\} \\
& \geq(1+o(1)) \sqrt{\Theta(w)}\left(\frac{\tilde{v}-\tilde{u}}{r}\right)^{2}
\end{aligned}
$$


for $r \leq r_{0}$, since $\cos (2 w)$ is close to 1 for small $r_{0}$. In addition,

$$
\begin{aligned}
(I V)_{b} & \equiv \frac{1}{\sqrt{\Theta(w)}}\left(\frac{\tilde{v}-\tilde{u}}{r}\right)^{2}\left\{-\frac{2 w_{r} \sin (2 w)}{r}+\frac{\sin ^{2}(2 w)}{r^{2}}\right\} \\
& =\left(-2 w_{r}+\frac{2 w}{r}\right) \frac{\sin (2 w)}{r \sqrt{\Theta(w)}\left(\frac{\tilde{v}-\tilde{u}}{r}\right)^{2}+o(1)\left(\frac{\tilde{v}-\tilde{u}}{r}\right)^{2}} \\
& \geq o(1)\left(\frac{\tilde{v}-\tilde{u}}{r}\right)^{2} .
\end{aligned}
$$

Furthermore,

$$
\begin{aligned}
(I V)_{c} & \equiv \frac{2 w_{r}}{\sqrt{\Theta(w)}}\left(\tilde{v}_{r}-\tilde{u}_{r}\right)\left(\frac{\tilde{v}-\tilde{u}}{r}\right)\left(\frac{\sin (2 w)}{r}-w_{r}\right) \\
& \geq\left(w_{r}+o(1)\right) \frac{2 w_{r}}{\sqrt{\Theta(w)}}\left(\tilde{v}_{r}-\tilde{u}_{r}\right)\left(\frac{\tilde{v}-\tilde{u}}{r}\right) \\
& \geq-\left(\frac{w_{r}^{2}}{\sqrt{\Theta(w)}}+o(1)\right)\left[0.9\left(\tilde{v}_{r}-\tilde{u}_{r}\right)^{2}+\frac{1}{0.9}\left(\frac{\tilde{v}-\tilde{u}}{r}\right)^{2}\right] .
\end{aligned}
$$

Now, with $\Theta(w)=3 w_{r}^{2}+o(1)$,

$$
\begin{aligned}
I V & =(I V)_{a}+(I V)_{b}+(I V)_{c}+w_{r}^{2}\left(\tilde{v}_{r}-\tilde{u}_{r}\right)^{2} / \sqrt{\Theta(w)}+\beta(\tilde{v}-\tilde{u})^{2} \\
& \geq\left(0.1 \frac{w_{r}^{2}}{\sqrt{\Theta(w)}}+o(1)\right)\left(\tilde{v}_{r}-\tilde{u}_{r}\right)^{2}+\left(\sqrt{\Theta(w)}-\frac{1}{0.9} \frac{w_{r}^{2}}{\sqrt{\Theta(w)}}+o(1)\right)\left(\frac{\tilde{v}-\tilde{u}}{r}\right)^{2} \\
& \geq\left(\frac{1.7 w_{r}^{2}}{0.9 \sqrt{\Theta(w)}}+o(1)\right)\left(\frac{\tilde{v}-\tilde{u}}{r}\right)^{2} \\
& \geq\left(\frac{1.7}{0.9 \sqrt{3}} \frac{\sqrt{\delta_{1}}}{4}+o(1)\right)(\tilde{v}-\tilde{u})^{2} \\
& \geq \frac{1}{10} \sqrt{\delta_{1}}(\tilde{v}-\tilde{u})^{2}
\end{aligned}
$$

for $r \leq r_{0}$, provided $r_{0}$ is sufficiently small.

With $r_{0}$ being fixed now, we will focus on $r \in\left[r_{0}, 1\right]$. Employing the CauchySchwarz inequality, and $|\sin w /(r \sqrt{\Theta(w)})| \leq 1$, we have

$$
\begin{aligned}
\left|\frac{2 w_{r} \sin (2 w)}{r^{2} \sqrt{\Theta(w)}}(\tilde{v}-\tilde{u})\left(\tilde{v}_{r}-\tilde{u}_{r}\right)\right| & \leq \frac{1}{\sqrt{\Theta(w)}}\left[w_{r}^{2}\left(\tilde{v}_{r}-\tilde{u}_{r}\right)^{2} / 10+10 \sin ^{2}(2 w)(\tilde{v}-\tilde{u})^{2} / r^{4}\right] \\
& \leq \frac{1}{10 \sqrt{\Theta(w)}} w_{r}^{2}\left(\tilde{v}_{r}-\tilde{u}_{r}\right)^{2}+40(\tilde{v}-\tilde{u})^{2} / r_{0}^{3} .
\end{aligned}
$$

Moreover, we have three other inequalities:

$$
\begin{aligned}
\left|\frac{2}{r}\left(\sqrt{\Theta(w)}+\frac{w_{r}^{2}}{\sqrt{\Theta(w)}}\right)(\tilde{v}-\tilde{u})\left(\tilde{v}_{r}-\tilde{u}_{r}\right)\right| \leq & \frac{1}{10}\left(\sqrt{\Theta(w)}+\frac{w_{r}^{2}}{\sqrt{\Theta(w)}}\right)\left(\tilde{v}_{r}-\tilde{u}_{r}\right)^{2} \\
& +\frac{10}{r_{0}^{2}}\left(\sqrt{\Theta(w)}+\frac{w_{r}^{2}}{\sqrt{\Theta(w)}}\right)(\tilde{v}-\tilde{u})^{2}, \\
\left|\frac{2 w_{r} \sin (2 w)}{r^{3} \sqrt{\Theta(w)}}(\tilde{v}-\tilde{u})^{2}\right| & \leq \frac{4}{r_{0}^{3}}(\tilde{v}-\tilde{u})^{2},
\end{aligned}
$$

and

$$
\left|\frac{2 \sqrt{\Theta(w)} \cos (2 w)}{r^{2}}(\tilde{v}-\tilde{u})^{2}\right| \leq \frac{2 \sqrt{\Theta(w)}}{r_{0}^{2}}(\tilde{v}-\tilde{u})^{2} .
$$


We note that $\left(\sqrt{\Theta(w)}+\frac{w_{r}^{2}}{\sqrt{\Theta(w)}}\right)$ is a bounded quantity. Now pick sufficiently large $\beta>0$, which depends on $r_{0}$, such that for $r \geq r_{0}$,

$$
I V \geq\left(1+\sqrt{\delta_{1}}\right)(\tilde{v}-\tilde{u})^{2} .
$$

Hence $\beta$ is now fixed.

Putting (3.11) and (3.12) into the double integral term in (3.7), we have

$$
\begin{aligned}
\int_{Q_{T}^{+}} d r d t \int_{0}^{1}\{I V\} d \xi & \geq \frac{\sqrt{\delta_{1}}}{10} \int_{Q_{T}^{+}}(\tilde{v}-\tilde{u})^{2} d r d t \\
& \geq \frac{\sqrt{\delta_{1}}}{10} e^{-2 \beta T_{0}} \int_{Q_{T}^{+}}(v-u)^{2} d r d t .
\end{aligned}
$$

If $\left|Q_{T}^{+}\right| \neq 0$, then there exists a $\delta_{2}>0$ such that $\int_{Q_{T}^{+}}(v-u)^{2} d r d t \geq \delta_{2}$. This gives a contradiction to inequality (3.7). That $\left|Q_{T}^{+}\right|=0$ means $u \geq v$ in $\overline{Q_{T}}$. This completes the proof of case (i).

(ii) Second case.

We can repeat the same argument as in case (i) on the domain $[0,1] \times\left[0, T_{1}\right]$. Hence we conclude that $u \geq v$ in this domain. Thus $u_{r}\left(0, T_{1}\right) \geq v_{r}\left(0, t_{1}\right) \geq \delta_{1}$. But then this contradicts the definition of $T_{1}$. Hence this case does not exist.

We therefore complete the proof of Lemma 3.1.

\section{4. $m$-HARMONIC HEAT FLOW}

For any $\zeta \in C^{2}(0,1) \cap C[0,1]$, let

$$
\tau(\zeta) \equiv \zeta_{r r}+\frac{n-1}{r} \zeta_{r}+\left(\frac{n}{2}-1\right) \frac{\theta_{r}}{\theta} \zeta_{r}-(n-1) \frac{\sin \zeta \cos \zeta}{r^{2}},
$$

where $\theta=\Theta(\zeta) \equiv \zeta_{r}^{2}+(n-1) \frac{\sin ^{2} \zeta}{r^{2}}$. There is a one-parameter family of functions satisfying

$$
\tau(\phi)=0 \text { for } r \in(0,1), \quad \text { and } \quad \phi(0)=0,
$$

given by

$$
\phi=\phi(r, \lambda)=2 \tan ^{-1}\left(\frac{r}{\lambda}\right) .
$$

When $p=n$, (4.3) gives a family of steady states of (1.5) (44).

For any $r \in[0,1]$ and $\lambda \in(0, \infty)$, it is clear that $0 \leq \phi(r, \lambda)<\pi$. Since

$$
\tan \left(\frac{\phi}{2}\right)=\frac{r}{\lambda}
$$

it follows that

$$
\phi_{r}=\frac{2 \lambda}{\lambda^{2}+r^{2}}=\frac{\sin \phi}{r} \geq 0
$$

and

$$
\phi_{r r}=\frac{\sin \phi}{r^{2}}(\cos \phi-1) \leq 0 .
$$

Thus $\phi$ is always increasing and concave in $r$. Moreover, $\phi$ increases as $\lambda$ decreases. This is due to the facts that $\phi \in C^{2+\alpha, 1+\alpha / 2}([0,1] \times(0, \infty))$ and $\partial \phi / \partial \lambda=-2 r /\left(\lambda^{2}+\right.$ $\left.r^{2}\right)<0$. Let $\Theta_{0}=\Theta(\phi)=\phi_{r}^{2}+(n-1) \frac{\sin ^{2} \phi}{r^{2}}$. Straightforward calculation yields

$$
\Theta_{0}=n \phi_{r}^{2}=\frac{n \sin ^{2} \phi}{r^{2}}
$$


and

$$
\frac{\left(\Theta_{0}\right)_{r}}{\Theta_{0}}=\frac{2(\cos \phi-1)}{r} .
$$

We will specify $\lambda$ as a $C^{\infty}$ function of $t$ in $[0, T)$ for some $T>0$ shortly. Moreover, let $\gamma=3-\epsilon$, where $\epsilon$ is a small positive number which will be determined later. Set

$$
v(r, t)=\phi(r, \lambda(t))+\epsilon r^{\gamma} .
$$

Clearly, $v$ is in $C^{2+\alpha, \frac{2+\alpha}{2}}([0,1] \times[0, T))$.

We now specify the choice of $\lambda$ as a function of $t$. Take any $\lambda_{0}>0$. Let $\lambda$ satisfy the initial value problem

$$
\begin{aligned}
& \frac{d \lambda}{d t}=-\delta \lambda^{\omega}, \\
& \lambda(0)=\lambda_{0},
\end{aligned}
$$

for some $\delta>0$ and $0<\omega<1$. Thus $\partial v / \partial t>0$. (We note that $\omega$ here is to be chosen later. In 2 the authors picked $\omega=\gamma-1$ in equation (1.7). We need a different value of $\omega$ in our case.)

First we will show that the derivative of $v$ goes to infinity in finite time. A simple calculation gives

$$
v_{r}(0, t)=\phi_{r}(0, \lambda(t))=\frac{2}{\lambda(t)} .
$$

From (4.5), $\lambda$ decays monotonically from its initial value $\lambda_{0}$ to 0 at a finite time $T$; indeed,

$$
\lambda(t)=\left\{\lambda_{0}^{1-\omega}-(1-\omega) \delta t\right\}^{\frac{1}{1-\omega}},
$$

and

$$
T=\frac{\lambda_{0}^{1-\omega}}{(1-\omega) \delta} .
$$

Therefore, $v(r, \cdot)$ increases with $t$, and as $t \uparrow T, v_{r}(0, t)$ goes to infinity.

To prove Theorem 1.3, we need to show that $v$ is a subsolution. In order to do that, we have a key lemma for the estimate of $\tau(v)$, the proof of which will be postponed to the next section.

Lemma 4.1. Let $v$ be defined as (4.4). For $n=p=3$ and $\gamma=3-\epsilon$, if $\epsilon$ is sufficiently small, then $\tau(v)>2 \epsilon r^{\gamma-2}$.

Theorem 4.1. For $p=n=3$ and $\left|\varphi_{0}(1)\right|>\pi$, if $\epsilon$ is sufficiently small, then $\gamma, \omega$, and $\delta$ can be chosen as functions of $\epsilon$ so that $v$ is a subsolution of (1.5) and (1.6).

Proof. Let $\Theta(v) \equiv v_{r}^{2}+(n-1) \sin ^{2} v / r^{2}$. By Lemma 4.1. for any $0<r<1$ and $0<t<T$,

$$
\begin{aligned}
\mathcal{L} v & \equiv \Theta(v)^{1 / 2} \tau(v)-\frac{\partial v}{\partial t} \\
& >2 \epsilon r^{\gamma-2} \Theta(v)^{1 / 2}-\frac{2 \delta r \lambda^{\omega}}{\lambda^{2}+r^{2}} \\
& =2 r^{\gamma-2}\left\{\epsilon \Theta(v)^{1 / 2}-\frac{\delta r^{3-\gamma} \lambda^{\omega}}{\lambda^{2}+r^{2}}\right\}
\end{aligned}
$$


Given $\epsilon>0$, choosing $\omega \in\left(1-\frac{\epsilon}{4-\epsilon}, 1\right)$, and using Young's inequality, we have

$$
\begin{aligned}
\frac{r^{3-\gamma} \lambda^{\omega}}{\lambda^{2}+r^{2}} & =r^{3-\gamma}\left(\frac{\lambda}{\lambda^{2}+r^{2}}\right)^{\omega}\left(\lambda^{2}+r^{2}\right)^{\omega-1} \\
& \leq\left(\frac{\lambda}{\lambda^{2}+r^{2}}\right)^{\omega} r^{3-\gamma-2(1-\omega)} \\
& =\left(\frac{\sin \phi}{2 r}\right)^{\omega} r^{\epsilon-2(1-\omega)} \\
& \leq \frac{1}{2^{\omega}}\left\{\omega\left(\frac{\sin \phi}{r}\right)+(1-\omega) r^{\frac{\epsilon}{1-\omega}-2}\right\} \\
& \leq \frac{1}{2^{\omega}}\left\{\omega\left(\frac{\sin \phi}{r}\right)+(1-\omega) r^{\gamma-1}\right\} \\
& \leq \frac{\sin \phi}{r}+r^{\gamma-1} .
\end{aligned}
$$

Recall that

$$
\Theta(v)^{1 / 2} \geq v_{r}=\frac{\sin \phi}{r}+\epsilon \gamma r^{\gamma-1}
$$

If $\delta$ is sufficiently small, then $\mathcal{L} v>0$ for any $0<r<1$, and $0<t<T$. Thus we have shown that $v$ is a strict subsolution. (We will check the condition that $v(1, t) \leq \varphi(1, t)=\varphi_{0}(1)$ below. $)$

Proof of Theorem 1.3. Let the initial condition $u_{0}$ be of the form in (1.3) and $\left|\varphi_{0}(1)\right|>\pi$. Without loss of generality, we may assume $\varphi_{0}(1)>\pi$ (for otherwise we consider $-\varphi$, which is also a solution to (1.5) and (1.6)). The constant function $2 k \pi+0.1$ is a strict upper solution of (1.5), where the positive integer $k$ is chosen so that $\varphi_{0}(1)$ is less than this constant function. Hence $\varphi$ is uniform bounded as long as the solution exists.

On the other hand, taking $\epsilon$ sufficiently small,

$$
v(1, t)=\phi(1, \lambda(t))+\epsilon<\varphi_{0}(1)
$$

Thus $v$ is a weak lower solution for (1.5) and (1.6). Also,

$$
v_{r}(0, t)=\frac{2}{\lambda(t)} \geq \frac{2}{\lambda_{0}}
$$

By choosing $\lambda_{0}$ sufficiently large, we can also ensure that the initial condition $\varphi_{0} \geq v$. Therefore, we apply Lemma 3.1 to conclude $\varphi \geq v$. Since $v_{r}$ goes to infinity in finite time, so does $\varphi_{r}$. Recalling that $|\nabla u|^{2}=\varphi_{r}^{2}+(n-1) \sin ^{2} \varphi / r^{2}$, we therefore have shown the solution of (1.1) and (1.2) is to blow up in finite time. 


\section{Proof of Lemma 4.1}

Recall $\Theta_{0}=n \sin ^{2} \phi / r^{2}$. Define

$$
\begin{aligned}
\Theta(v) \equiv & v_{r}^{2}+(n-1) \frac{\sin ^{2} v}{r^{2}} \\
= & \frac{\sin ^{2} \phi}{r^{2}}+2 \epsilon \gamma r^{\gamma-2} \sin \phi+\epsilon^{2} \gamma^{2} r^{2 \gamma-2} \\
& +\frac{n-1}{r^{2}}\left(\sin \phi \cos \left(\epsilon r^{\gamma}\right)+\cos \phi \sin \left(\epsilon r^{\gamma}\right)\right)^{2} \\
= & \frac{\sin ^{2} \phi}{r^{2}}+2 \epsilon \gamma r^{\gamma-2} \sin \phi+\epsilon^{2} \gamma^{2} r^{2 \gamma-2} \\
& +\frac{n-1}{r^{2}}\left(\sin ^{2} \phi \cos ^{2}\left(\epsilon r^{\gamma}\right)+\sin \phi \cos \phi \sin \left(2 \epsilon r^{\gamma}\right)+\cos ^{2} \phi \sin ^{2}\left(\epsilon r^{\gamma}\right)\right) \\
= & \frac{n \sin ^{2} \phi}{r^{2}}+2 \epsilon \gamma r^{\gamma-2} \sin \phi+\epsilon^{2} \gamma^{2} r^{2 \gamma-2}-\frac{(n-1)}{r^{2}} \sin ^{2} \phi \sin ^{2}\left(\epsilon r^{\gamma}\right) \\
& +\frac{n-1}{r^{2}} \sin \phi \cos \phi \sin \left(2 \epsilon r^{\gamma}\right)+\frac{n-1}{r^{2}} \cos ^{2} \phi \sin ^{2}\left(\epsilon r^{\gamma}\right) \\
= & \Theta_{0}+2 \epsilon r^{\gamma-2} \sin \phi(\gamma+(n-1) \cos \phi) \\
& +\epsilon^{2} r^{2 \gamma-2}\left[\gamma^{2}+(n-1) \cos 2 \phi\right]+O\left(\epsilon^{3} r^{3 \gamma-2}\right) .
\end{aligned}
$$

Now, we want to find $\Theta_{r}$. From (5.1),

$$
\begin{aligned}
\Theta(v)_{r}= & \left(\Theta_{0}\right)_{r}+2 \epsilon \gamma\left((\gamma-2) r^{\gamma-3} \sin \phi+r^{\gamma-2} \phi_{r} \cos \phi\right)+2(\gamma-1) \epsilon^{2} \gamma^{2} r^{2 \gamma-3} \\
& +\frac{n-1}{2} \frac{\partial}{\partial r}\left(\frac{\sin 2 \phi \sin \left(2 \epsilon r^{\gamma}\right)}{r^{2}}\right)+(n-1) \frac{\partial}{\partial r}\left(\frac{\cos 2 \phi \sin ^{2}\left(\epsilon r^{\gamma}\right)}{r^{2}}\right)
\end{aligned}
$$

Let

$$
\begin{aligned}
A_{1} \equiv & \frac{\partial}{\partial r}\left(\frac{\sin 2 \phi \sin \left(2 \epsilon r^{\gamma}\right)}{r^{2}}\right) \\
= & \frac{2 \cos 2 \phi \sin \left(2 \epsilon r^{\gamma}\right)}{r^{2}} \phi_{r}+\frac{\sin 2 \phi \cos \left(2 \epsilon r^{\gamma}\right)}{r^{2}} 2 \epsilon \gamma r^{\gamma-1}-\frac{2 \sin 2 \phi \sin \left(2 \epsilon r^{\gamma}\right)}{r^{3}} \\
= & 4 \epsilon r^{\gamma-3} \cos 2 \phi \sin \phi\left(1+O\left(\epsilon^{2} r^{2 \gamma}\right)\right)+2 \epsilon \gamma r^{\gamma-3} \sin 2 \phi\left(1+O\left(\epsilon^{2} r^{2 \gamma}\right)\right) \\
& -4 \epsilon r^{\gamma-3} \sin 2 \phi\left(1+O\left(\epsilon^{2} r^{2 \gamma}\right)\right) \\
= & 4 \epsilon r^{\gamma-3} \sin \phi[\cos 2 \phi+(\gamma-2) \cos \phi]+O\left(\epsilon^{3} r^{3 \gamma-3}\right),
\end{aligned}
$$

and

$$
\begin{aligned}
A_{2} \equiv & \frac{\partial}{\partial r}\left(\frac{\cos 2 \phi \sin ^{2}\left(\epsilon r^{\gamma}\right)}{r^{2}}\right) \\
= & -\frac{\sin 2 \phi \sin ^{2}\left(\epsilon r^{\gamma}\right)}{r^{2}} 2 \frac{\sin \phi}{r}+\frac{\cos 2 \phi \sin \left(\epsilon r^{\gamma}\right) \cos \left(\epsilon r^{\gamma}\right)}{r^{2}} 2 \epsilon \gamma r^{\gamma-1} \\
& -\frac{2 \cos 2 \phi \sin ^{2}\left(\epsilon r^{\gamma}\right)}{r^{3}} \\
= & \epsilon^{2} r^{2 \gamma-3}[-2 \sin \phi \sin 2 \phi+2 \gamma \cos 2 \phi-2 \cos 2 \phi]+O\left(\epsilon^{4} r^{4 \gamma-3}\right) .
\end{aligned}
$$


Therefore,

$$
\begin{aligned}
\Theta(v)_{r}= & \left(\Theta_{0}\right)_{r}+2 \epsilon \gamma r^{\gamma-3} \sin \phi(\gamma-2+\cos \phi)+2(\gamma-1) \epsilon^{2} \gamma^{2} r^{2 \gamma-3} \\
& +2(n-1) \epsilon r^{\gamma-3} \sin \phi[\cos 2 \phi+(\gamma-2) \cos \phi] \\
& +(n-1) \epsilon^{2} r^{2 \gamma-3}[-2 \sin \phi \sin 2 \phi+2 \gamma \cos 2 \phi-2 \cos 2 \phi]+O\left(\epsilon^{3} r^{3 \gamma-3}\right) \\
= & \left(\Theta_{0}\right)_{r}+2 \epsilon r^{\gamma-3} \sin \phi[\gamma(\gamma-2+\cos \phi)+(n-1)(\cos 2 \phi+(\gamma-2) \cos \phi)] \\
& +2 \epsilon^{2} r^{2 \gamma-3}\left[\gamma^{2}(\gamma-1)+(n-1)\left((\gamma-1) \cos 2 \phi-2 \sin ^{2} \phi \cos \phi\right)+O\left(\epsilon r^{\gamma}\right)\right] .
\end{aligned}
$$

Next, we define the operator $\tilde{\tau}$ such that for any smooth $w$,

$$
\tilde{\tau}(w) \equiv w_{r r}+\frac{n-1}{r} w_{r}+\frac{1}{2} \frac{\Theta(w)_{r}}{\Theta(w)} w_{r}-\frac{n-1}{r^{2}} \sin w \cos w .
$$

Fix $p=3$ from now on. We note that the right-hand side of (1.5) is then $\Theta(\varphi)^{\frac{1}{2}} \tilde{\tau}(\varphi)$. We now estimate $\tilde{\tau}(v)$ as follows:

$$
\begin{aligned}
\Theta(v)^{\frac{1}{2}} \tilde{\tau}(v)=\Theta(v)^{\frac{1}{2}}\left[v_{r r}+\frac{n-1}{r} v_{r}+\frac{\Theta(v)_{r}}{2 \Theta(v)} v_{r}-\frac{n-1}{2 r^{2}} \sin 2 v\right] \\
=\Theta(v)^{\frac{1}{2}}\left[\frac{\sin \phi}{r^{2}}(\cos \phi+n-2)+\gamma \epsilon r^{\gamma-2}(\gamma+n-2)\right. \\
\left.-\frac{n-1}{2 r^{2}} \sin 2 v+\frac{\Theta(v)_{r}}{2 \Theta(v)} v_{r}\right] \\
=\Theta(v)^{\frac{1}{2}}\left[\frac{(n-1)(\sin 2 \phi-\sin 2 v)}{2 r^{2}}+\frac{(n-2) \sin \phi(1-\cos \phi)}{r^{2}}\right. \\
\left.+\gamma \epsilon r^{\gamma-2}(\gamma+n-2)+\frac{\Theta(v)_{r}}{2 \Theta(v)} v_{r}\right] \\
\geq \Theta(v)^{\frac{1}{2}}\left\{\frac{(n-2) \sin \phi(1-\cos \phi)}{r^{2}}+\epsilon r^{\gamma-2}[\gamma(\gamma+n-2)-n+1]\right. \\
\left.\quad+\frac{\Theta(v)_{r}}{2 \Theta(v)} v_{r}\right\},
\end{aligned}
$$

because

$$
\begin{aligned}
\sin 2 \phi-\sin 2 v & =2 \sin (\phi-v) \cos (\phi+v) \\
& =-2 \sin \epsilon r^{\gamma} \cos (\phi+v) \\
& \geq-2 \epsilon r^{\gamma} .
\end{aligned}
$$

Putting in the estimate for $\Theta(v)_{r}$, we have

$$
\begin{aligned}
& \Theta(v)^{1 / 2} \tilde{\tau}(v) \geq \Theta(v)^{1 / 2} \epsilon r^{\gamma-2}[\gamma(\gamma+n-2)-n+1] \\
& +(n-2) \Theta(v)^{1 / 2} \frac{\sin \phi(1-\cos \phi)}{r^{2}} \\
& +\frac{1}{2} \Theta(v)^{-1 / 2}\left(\frac{\sin \phi}{r}+\epsilon \gamma r^{\gamma-1}\right)\left\{\frac{2 n \sin ^{2} \phi(\cos \phi-1)}{r^{3}}\right. \\
& +2 \epsilon r^{\gamma-3} \sin \phi[\gamma(\gamma-2+\cos \phi)+(n-1)(\cos 2 \phi+(\gamma-2) \cos \phi)] \\
& +2 \epsilon^{2} r^{2 \gamma-3}\left[\gamma^{2}(\gamma-1)+(n-1)(\gamma-1) \cos 2 \phi-2(n-1) \sin ^{2} \phi \cos \phi\right. \\
& \left.\left.+O\left(\epsilon r^{\gamma}\right)\right]\right\} .
\end{aligned}
$$


When $n=3$ with $\gamma=3-\epsilon$ for small $\epsilon>0$, we can conclude from (5.1) that

$$
\Theta(v) \geq \frac{3 \sin ^{2} \phi}{r^{2}}
$$

consequently, the terms

$$
\begin{gathered}
(n-2) \Theta(v)^{1 / 2} \frac{\sin \phi(1-\cos \phi)}{r^{2}}+\Theta(v)^{-1 / 2} n \frac{\sin ^{3} \phi}{r^{4}}(\cos \phi-1) \\
=\Theta(v)^{-1 / 2} \frac{\sin \phi(1-\cos \phi)}{r^{2}}\left[(n-2) \Theta(v)-n \frac{\sin ^{2} \phi}{r^{2}}\right] \geq 0 .
\end{gathered}
$$

Moreover,

$$
\begin{aligned}
& \epsilon^{3} \gamma r^{3 \gamma-4}\left[\gamma^{2}(\gamma-1)+(n-1)(\gamma-1) \cos 2 \phi-2(n-1) \sin ^{2} \phi \cos \phi+O\left(\epsilon r^{\gamma}\right)\right] \\
& \quad \geq \epsilon^{3} \gamma r^{3 \gamma-4}\left[\gamma^{2}(\gamma-1)-(n-1)(\gamma-1)-2(n-1)+O(\epsilon)\right] \\
& \quad>0
\end{aligned}
$$

The terms in both (5.7) and (5.8) are positive and can be dropped in (5.5), which then gives

$$
\begin{aligned}
\Theta(v)^{1 / 2} \tilde{\tau}(v) \geq & \Theta(v)^{1 / 2} \epsilon r^{\gamma-2}[\gamma(\gamma+n-2)-n+1] \\
& +\Theta(v)^{-1 / 2}\left\{\epsilon r^{\gamma-4} \sin ^{2} \phi[\gamma(\gamma-n-2)\right. \\
& +(2 n \gamma-2 n+2) \cos \phi+(n-1) \cos 2 \phi] \\
& +\epsilon^{2} r^{2 \gamma-4} \sin \phi\left[\gamma^{2}(2 \gamma-3)+(n-1)(2 \gamma-1) \cos 2 \phi\right. \\
& \left.\left.+\left((n-1)(\gamma-2) \gamma-2(n-1) \sin ^{2} \phi+\gamma^{2}\right) \cos \phi+O\left(\epsilon r^{\gamma}\right)\right]\right\} .
\end{aligned}
$$

Observe that

$$
(2 n \gamma-2 n+2) \cos \phi+(n-1) \cos 2 \phi \geq-(2 n \gamma-2 n+2)-(n-1)
$$

and

$$
\begin{aligned}
& \gamma^{2}(2 \gamma-3)+(n-1)(2 \gamma-1) \cos 2 \phi \\
& \quad+\left((n-1)(\gamma-2) \gamma-2(n-1) \sin ^{2} \phi+\gamma^{2}\right) \cos \phi \\
& \geq \gamma^{2}(2 \gamma-3)+(n-1)(2 \gamma-1)\left(1-2 \sin ^{2} \phi\right) \\
&-\left((n-1)(\gamma-2) \gamma-2(n-1) \sin ^{2} \phi+\gamma^{2}\right) \\
&= \gamma^{2}(2 \gamma-4)+(n-1)\left(4 \gamma-1-\gamma^{2}\right)-4(\gamma-1)(n-1) \sin ^{2} \phi \\
& \geq \gamma^{2}(2 \gamma-4)+(n-1)\left(3-\gamma^{2}\right) ;
\end{aligned}
$$


hence, we have

$$
\begin{aligned}
\Theta(v)^{1 / 2} \tilde{\tau}(v) \geq \epsilon & r^{\gamma-2} \Theta(v)^{-1 / 2}\{\Theta(v)[\gamma(\gamma+n-2)-n+1] \\
& +\frac{\sin ^{2} \phi}{r^{2}}\left[\gamma^{2}-3 n \gamma-2 \gamma+n-1\right] \\
\left.+\epsilon r^{\gamma-2} \sin \phi\left[\gamma^{2}(2 \gamma-4)+(n-1)\left(3-\gamma^{2}\right)+O\left(\epsilon r^{\gamma}\right)\right]\right\} & \\
= & \epsilon r^{\gamma-2} \Theta(v)^{-1 / 2}\left\{(2 n+4+O(\epsilon)) \Theta(v)+(-8 n+2+O(\epsilon)) \frac{\sin ^{2} \phi}{r^{2}}\right. \\
+ & \left.(24-6 n+O(\epsilon)) \epsilon r^{\gamma-2} \sin \phi\right\},
\end{aligned}
$$

with $\gamma=3-\epsilon$.

Recall we have already set $n=3$; thus,

$$
\begin{array}{cc}
\Theta(v)^{1 / 2} \tilde{\tau}(v) \geq \epsilon r^{\gamma-2} \Theta(v)^{-1 / 2}\{( & (10+O(\epsilon)) \Theta(v)+(-22+O(\epsilon)) \frac{\sin ^{2} \phi}{r^{2}} \\
& \left.+(6+O(\epsilon)) \epsilon r^{\gamma-2} \sin \phi\right\} \\
\geq 2 \epsilon r^{\gamma-2} \Theta(v)^{1 / 2}, &
\end{array}
$$

after we have invoked (5.6). Since $\tau(v)=\tilde{\tau}(v)$ when $n=p=3$, the proof of Lemma 4.1 is complete.

\section{Appendix 1: Derivation of an equation for $b_{1}$}

With the form of $u_{3}$ from equation (2.5), the third equation in (1.1) becomes

$$
\begin{aligned}
\frac{\partial}{\partial t}\left(\sin a \cos \left(\beta+b_{1}\right)\right)= & \operatorname{div}\left[|\nabla u| \nabla\left(\sin a \cos \left(\beta+b_{1}\right)\right)\right] \\
& +|\nabla u|^{3} \sin a \cos \left(\beta+b_{1}\right) .
\end{aligned}
$$

In this appendix, we will discover the form of this equation in spherical coordinates when $\beta=\pi / 2$. Note that the local existence result in section 2 implies that $u$ is in $C^{2,1}\left(\overline{D^{3}} \times[0, \delta]\right)$ for some time $\delta>0$.

Let $v=\sin a \sin b_{1}$. Our first concern is the differentiability of $v$ in the spherical coordinates. We know from section 2 that $v$ is a function of $(r, t)$. Moreover, $v$ is in $C([0,1] \times[0, \delta])$ and is $C^{2,1}$ whenever $\sin a \neq 0$ and $r \neq 0$. Under such conditions, we can derive the governing parabolic equation on $v$. The following are calculation details.

(i) The term on the left-hand side of (6.1) is

$$
\left.\frac{\partial}{\partial t}\left(\sin a \cos \left(\beta+b_{1}\right)\right)\right|_{\beta=\pi / 2}=-\frac{\partial v}{\partial t} .
$$

(ii) The last term on the right-hand side of (6.1) is

$$
\left.|\nabla u|^{3} \sin a \cos \left(\beta+b_{1}\right)\right|_{\beta=\pi / 2}=-\left.|\nabla u|^{3}\right|_{\beta=\pi / 2} v .
$$


(iii) The first term on the right-hand side of (6.1) can be computed as follows:

$$
\begin{aligned}
\left.\mathcal{T} \equiv \operatorname{div}\left[|\nabla u| \nabla\left(\sin a \cos \left(\beta+b_{1}\right)\right)\right]\right|_{\beta=\pi / 2} \\
=\left.\left\{|\nabla u| \Delta\left(\sin a \cos \left(\beta+b_{1}\right)\right)+\nabla(|\nabla u|) \cdot \nabla\left(\sin a \cos \left(\beta+b_{1}\right)\right)\right\}\right|_{\beta=\pi / 2} \\
=\left\{| \nabla u | \left[-\sin b_{1} \Delta(\sin a)-\sin \left(\beta+b_{1}\right) \sin a\left(\Delta \beta+\Delta b_{1}\right)\right.\right. \\
\quad \quad-\sin a \cos \left(\beta+b_{1}\right)\left(\nabla \beta+\nabla b_{1}\right) \cdot\left(\nabla \beta+\nabla b_{1}\right) \\
\left.\quad-2 \cos a \cos b_{1} \nabla a \cdot\left(\nabla \beta+\nabla b_{1}\right)\right] \\
\left.\quad \quad+\nabla(|\nabla u|) \cdot \nabla\left(\sin a \cos \left(\beta+b_{1}\right)\right)\right\}\left.\right|_{\beta=\pi / 2} \\
=\left\{| \nabla u | \left[-\sin b_{1} \Delta(\sin a)-\cos b_{1} \sin a \Delta b_{1}+\sin a \sin b_{1}\left(|\nabla \beta|^{2}+\left|\nabla b_{1}\right|^{2}\right)\right.\right. \\
\left.\left.\quad-2 \cos a \cos b_{1} \nabla a \cdot \nabla b_{1}\right]+\nabla(|\nabla u|) \cdot \nabla\left(\sin a \cos \left(\beta+b_{1}\right)\right)\right\}\left.\right|_{\beta=\pi / 2},
\end{aligned}
$$

with the last equality following from the facts that $\left.\Delta \beta\right|_{\beta=\pi / 2}=0, \nabla \beta \cdot \nabla a=0$, and $\nabla \beta \cdot \nabla b_{1}=0$. To derive an equation in $v$, we can rewrite

$$
\begin{aligned}
\mathcal{T} & =\left.\left\{|\nabla u|\left(-\Delta v+|\nabla \beta|^{2} v\right)+\nabla(|\nabla u|) \cdot \nabla\left(\sin a \cos \left(\beta+b_{1}\right)\right)\right\}\right|_{\beta=\pi / 2} \\
& =\left\{|\nabla u|\left(-\Delta v+\frac{v}{r^{2}}\right)-\nabla(|\nabla u|) \cdot \nabla v-\left.\sin a \cos b_{1} \nabla(|\nabla u|) \cdot \nabla \beta\right|_{\beta=\pi / 2}\right.
\end{aligned}
$$

by making use of $|\nabla \beta|^{2}=1 / r^{2}$. A somewhat tedious calculation yields

$|\nabla u|^{2}=\left(a^{\prime}\right)^{2}+\sin ^{2} a\left[\left(b_{1}^{\prime}\right)^{2}+\left(c_{1}^{\prime}\right)^{2} \sin ^{2}\left(\beta+b_{1}\right)+1 / r^{2}+\sin ^{2}\left(\beta+b_{1}\right) /\left(r^{2} \sin ^{2} \beta\right)\right]$,

where the prime denotes the derivative with respect to $r$. Then it can be easily checked that

$$
\begin{aligned}
\left.\{\nabla(|\nabla u|) \cdot \nabla \beta\}\right|_{\beta=\pi / 2} & =\left.\frac{1}{r^{2}} \frac{\partial}{\partial \beta}(|\nabla u|)\right|_{\beta=\pi / 2} \\
& =-\left.\left\{\sin a \cos b_{1}\left(\left(c_{1}^{\prime}\right)^{2}+1 / r^{2}\right) v /\left(r^{2}|\nabla u|\right)\right\}\right|_{\beta=\frac{\pi}{2}}
\end{aligned}
$$

Therefore,

$$
\begin{aligned}
\mathcal{T}=\{\mid & \nabla u \mid\left(-\Delta v+\frac{v}{r^{2}}\right)-\nabla(|\nabla u|) \cdot \nabla v \\
& \left.+\frac{1}{r^{2}} \sin ^{2} a \cos ^{2} b_{1}\left(\left(c_{1}^{\prime}\right)^{2}+\frac{1}{r^{2}}\right)|\nabla u|^{-1} v\right\}\left.\right|_{\beta=\pi / 2} .
\end{aligned}
$$

Substituting (6.2)- (6.4) into (6.1) and letting $\beta=\pi / 2$, we get

$$
\begin{aligned}
\frac{\partial v}{\partial t}=\{ & |\nabla u| \Delta v+\frac{\partial(|\nabla u|)}{\partial r} \frac{\partial v}{\partial r} \\
& \left.\quad-\left[\frac{1}{r^{2}} \sin ^{2} a \cos ^{2} b_{1}\left(\left(c_{1}^{\prime}\right)^{2}+\frac{1}{r^{2}}\right)|\nabla u|^{-1}+\frac{|\nabla u|}{r^{2}}-|\nabla u|^{3}\right] v\right\}_{\beta=\pi / 2}
\end{aligned}
$$

With $\Delta v=v_{r r}+2 v_{r} / r,(6.5)$ can be viewed as

$$
v_{t}=A v_{r r}+B v_{r}+C v,
$$

where $A, B$ and $C$ are functions of $(r, t)$. Since $u \in C^{2,1}\left(\overline{D^{3}} \times[0, \delta]\right)$ and $|\nabla u| \neq 0$ at $t=0$, (6.6) remains uniformly parabolic with $C$ being bounded from above. This allows us to apply the maximum principle to (6.6). 


\section{Appendix 2: Derivation of an equation for $c_{1}$}

The derivation follows that in Appendix 1. After the derivation in appendix 1 and the argument in section 2 , we know $b_{1}=0$ already. Furthermore, we set $\beta=\pi / 2$ and $\gamma=0$. Let $\tilde{w}=\sin a \sin c_{1}$. With the form of $u_{2}$ from equation (2.5), the second equation in (1.1) then becomes

$$
\frac{\partial \tilde{w}}{\partial t}=\operatorname{div}\left[|\nabla u| \nabla\left(\sin a \sin \beta \sin \left(\gamma+c_{1}\right)\right]+|\nabla u|^{3} \tilde{w}\right.
$$

with the understanding that we have to evaluate at $\beta=\pi / 2$ and $\gamma=0$ on the right-hand side after taking the derivatives.

Provided $\sin a \neq 0$ and $r \neq 0, \tilde{w}$ as a function of $(r, t)$ is in $C^{2,1}$ so that we can carry out the differentiation in (7.1). The first term on the right-hand side of (7.1) can be computed as follows:

$$
\begin{aligned}
\mathcal{S} \equiv & \left.\operatorname{div}\left[|\nabla u| \nabla\left(\sin a \sin \beta \sin \left(\gamma+c_{1}\right)\right)\right]\right|_{\beta=\pi / 2, \gamma=0} \\
= & \left\{|\nabla u| \Delta\left(\sin a \sin \beta \sin \left(\gamma+c_{1}\right)\right)+\nabla(|\nabla u|)\right. \\
& \left.\quad \cdot \nabla\left(\sin a \sin \beta \sin \left(\gamma+c_{1}\right)\right)\right\}\left.\right|_{\beta=\pi / 2, \gamma=0} \\
= & \left\{| \nabla u | \left[\sin a \cos c_{1}\left(\Delta \gamma+\Delta c_{1}\right)-\sin a \sin c_{1}\left(|\nabla \gamma|^{2}+\left|\nabla c_{1}\right|^{2}\right)\right.\right. \\
& -\sin a \sin c_{1}|\nabla \beta|^{2}+\cos a \sin c_{1} \Delta a-\sin a \sin c_{1}|\nabla a|^{2} \\
& \left.+2 \cos a \cos c_{1} \nabla a \cdot \nabla c_{1}\right] \\
& \left.+\nabla(|\nabla u|) \cdot\left[\sin a \cos c_{1}\left(\nabla \gamma+\nabla c_{1}\right)+\cos a \sin c_{1} \nabla a\right]\right\}\left.\right|_{\beta=\pi / 2, \gamma=0} .
\end{aligned}
$$

Since $\left.\Delta \gamma\right|_{\beta=\pi / 2}=0, \nabla(|\nabla u|) \cdot \nabla \gamma=0$ and $|\nabla \beta|^{2}=\left\{|\nabla \gamma|^{2}\right\}_{\beta=\pi / 2}=1 / r^{2}$, we can further simplify the above term:

$$
\begin{aligned}
\mathcal{S}=\{ & |\nabla u|\left[\sin a \cos c_{1} \Delta c_{1}-\sin a \sin c_{1}\left(\frac{2}{r^{2}}+\left|\nabla c_{1}\right|^{2}\right)\right. \\
& \left.+\cos a \sin c_{1} \Delta a-\sin a \sin c_{1}|\nabla a|^{2}+2 \cos a \cos c_{1} \nabla a \cdot \nabla c_{1}\right] \\
& \left.+\nabla(|\nabla u|) \cdot\left[\sin a \cos c_{1} \nabla c_{1}+\cos a \sin c_{1} \nabla a\right]\right\}\left.\right|_{\beta=\pi / 2, \gamma=0} .
\end{aligned}
$$

Our aim is to assemble an equation in $\tilde{w}$; so we further manipulate to get

$$
\mathcal{S}=\left.\left\{|\nabla u|\left[\Delta \tilde{w}-\frac{2}{r^{2}} \tilde{w}\right]+\nabla(|\nabla u|) \cdot \nabla \tilde{w}\right\}\right|_{\beta=\pi / 2, \gamma=0} .
$$

Assembling all the information, with $b_{1}=0$ and setting $\beta=\pi / 2, \gamma=0$, the equation (7.1) will be transformed to

$$
\frac{\partial \tilde{w}}{\partial t}=\left\{|\nabla u| \Delta \tilde{w}+\frac{\partial(|\nabla u|)}{\partial r} \frac{\partial \tilde{w}}{\partial r}+\left[-\frac{2|\nabla u|}{r^{2}}+|\nabla u|^{3}\right] \tilde{w}\right\}_{\beta=\pi / 2, \gamma=0} .
$$

With $\Delta \tilde{w}=\tilde{w}_{r r}+2 \tilde{w}_{r} / r$, this equation is in the form of

$$
\tilde{w}_{t}=A \tilde{w}_{r r}+B \tilde{w}_{r}+C \tilde{w}
$$

with $A, B$ and $C$ functions of $(r, t)$. With solution $u$ being in $C^{2,1}\left(\overline{D^{3}} \times[0, \delta]\right)$ from the local existence result in section 2 and $|\nabla u| \neq 0$ at $t=0$, (17.3) remains uniformly parabolic with $C$ being bounded above. This allows application of the classical maximum principle.

\section{ACKNOWLEDGEMENTS}

The works of CNC and CKL are partially supported by the National Science Council, Taiwan, ROC. The works of LFC are partially supported by the Research Committee of The Hong Kong Polytechnic University (Grant No. G-T099). 


\section{REFERENCES}

[1] K.C. Chang and W.Y. Ding, A result on the global existence for heat flows of harmonic maps from $D^{2}$ into $S^{2}$, in "Nematics", J.M. Coron et al ed., Kluwer Academic Publishers (1990) 37-48. MR 94c:58048

[2] K.C. Chang, W.Y. Ding and R.G. Ye, Finite-time blow-up of the heat flow of harmonic maps from surfaces, J. Diff. Geometry, 36 (1992) 507-515. MR 93h:58043

[3] Y. Chen, M.C. Hong, N. Hungerbühler, Heat flow for p-harmonic maps with values into spheres, Math. Zeit. 215, 25-35, (1994). MR 94k:58145

[4] C.N. Chen, L.F. Cheung, Y.S. Choi and C.K. Law, Integrability of rotationally symmetric n-harmonic maps, preprint.

[5] C.N. Chen, L.F. Cheung, Y.S. Choi and C.K. Law, Asymptotic behaviours of rotationally symmetric harmonic maps and their heat flow, preprint.

[6] L.F. Cheung, C.K. Law, M.C. Leung and J.B. McLeod, Entire solutions of quasilinear differential equations corresponding to p-harmonic maps, Nonlinear Anal. T.M.A., 31 (1998) 701-715. MR 99g:58033

[7] R. Coron, J.-M. Ghidaglia, Explosion en temps fini pour le flot des applications harmoniques, C.R. Acad. Sci. Paris Sér. I 308, 339-344 (1989). MR 90g:58026

[8] W.Y. Ding, Lecture Notes on the Heat Flow of Harmonic Maps, NCTS, (1997).

[9] L.C. Evans, Partial regularity for stationary harmonic maps into spheres, Arch. Rat. Mech. Anal. 116, 101-113, (1991). MR 93m:5826

[10] J. Eells, J.H. Sampson, Harmonic mappings of Riemannian manifolds, Amer. J. Math. 86, 109-169 (1964) MR 29:1603

[11] M. Fuchs, The blow-up of p-harmonic maps, Manuscripta Math. 89-94, (1993). MR 94h:58060

[12] M. Giaquinta, E. Giusti, On the regularity of minima of variational integrals, Acta Math. 148, 31-40 (1982). MR 84b:58034

[13] D. Gilbarg and N. S. Trudinger; Elliptic partial differential equations of second order, 2nd edition, Springer-Verlag, 1983. MR 86c:35035

[14] J.F. Grotowski, Heat flow for harmonic maps, in "Nematics", J.M. Coron et al ed., Kluwer Academic Publishers (1990) 129-140. MR 98f:58042

[15] J.F. Grotowski, Finite time blow-up for the harmonic map heat flow, Calculus of Variations, 1 (1993) 231-236. MR 94k:58034

[16] Richard Hamilton, Harmonic Maps of Manifolds with Boundary, (1975), Lecture Notes in Mathematics, vol. 471, Springer-Verlag. MR 58:2872

[17] R. Hardt, D. Kinderlehrer, F.H. Lin, Mappings minimizing the $L^{p}$-norm of the gradient, Comm. Pure Appl. Math. 11, 555-588 (1987). MR 88k:58026

[18] F. Hélein, Regularité des applications faiblement harmoniques entre une surface et une varieté Riemannienne, C.R. Acad. Sci. Paris, Sér. I Math. 312, 591-596 (1991). MR 92e:58055

[19] S. Hildebrandt, H. Kaul, K.O. Widman, An existence theorem for harmonic mappings of Riemannian manifolds, Acta Math., 138, 1-16, (1977). MR 55:6478

[20] N. Hungerbühler, m-harmonic flow, Ann. Scuola Norm. Sup. Pisa Cl. Sci., 24, no. 4, 593-632 (1997). MR 99c:58046

[21] N.V. Krylov, Lectures on Elliptic and Parabolic Equations in Hölder Spaces, Graduate Studies in Math., Vol. 12, (1996), American Mathematical Society, Providence, RI. MR 97i:35001

[22] S. Luckhaus, Partial Hölder continuity for minima of certain energies among maps into a Riemannian manifold, Indiana Univ. Math. J. 37, 346-367, (1988). MR 89m:58043

[23] O.A. Ladyzenskaja, V.A. Solonnikov and N.N. Ural'ceva, Linear and Quasilinear Equations of Parabolic type, Translation of Mathematical Monographs, 23, (1967), American Mathematical Society, Providence, RI. MR 39:3159h

[24] N. Nakauchi, S. Takakuwa, A remark on p-harmonic maps, Nonlinear Anal., 25, 169-195, (1995). MR 96e:58046

[25] M. Rigoli, M. Salvatori and M. Vignati, Volume growth and p-subharmonic functions on complete manifolds, Math. Z. 227, no. 3, 357-375, (1998). MR 99h:58177

[26] Wilhelm Schlag, Schauder and $L^{p}$ estimates for parabolic systems via Campanato spaces, Comm. Partial Differential Equations, 21 (1996), pp. 1141-1175. MR 97k:35108

[27] P. Strzelecki, Regularity of p-harmonic maps from the p-dimensional ball into a sphere, Manuscripta Math. 82, 407-415, (1994). MR 95b:58047 
[28] J. Sacks, K. Uhlenbeck, The existence of minimal immersions of 2-spheres, Ann. of Math. 113, 1-24, (1981). MR 82f:58035

[29] R. Schoen, K. Uhlenbeck, A regularity theory for harmonic maps, J. Differential Geom., 17, 307-335, (1982). MR 84b:58037a Correction, J. Diff. Geom., 18, 329, (1983). MR 84b:58037b

[30] M. Struwe, On the evolution of harmonic mappings of Riemannian surfaces, Comment. Math. Helv. 60, 558-581 (1981). MR 87e:5856

Department of Mathematics, National Changhua University of Education, Changhua, Taiwan, Republic of China

E-mail address: chenc@math.ncue.edu.tw

Department of Applied Mathematics, The Hong Kong Polytechnic University, KowloOn, Hong Kong

E-mail address: matheclf@maun01.ma.polyu.edu.hk

Department of Mathematics, University of Connecticut, Storrs, Connecticut 062693009

E-mail address: choi@math.uconn.edu

Department of Applied Mathematics, National Sun Yat-Sen University, Kaohsiung, TAIWAN 80424, RePUBlic OF ChinA

E-mail address: law@math.nsysu.edu.tw 